\title{
Schwinger-Keldysh effective action for a relativistic Brownian particle in the AdS/CFT correspondence
}

\author{
Yanyan Bu๑ $\odot^{\dagger}$ and Biye Zhang $\odot^{*}$ \\ School of Physics, Harbin Institute of Technology, Harbin 150001, China
}

(Received 23 August 2021; accepted 30 August 2021; published 1 October 2021)

\begin{abstract}
We compute Schwinger-Keldysh effective action for a relativistic heavy quark (with constant background velocity) in strongly coupled $\mathcal{N}=4$ supersymmetric Yang-Mills plasma. The holographic dual description involves a noisy trailing string moving in Schwarzschild-AdS $\mathrm{S}_{5}$ black brane. The noise is caused by Hawking radiation emitted from string world sheet horizon. Besides quadratic terms, the effective action contains cubic interactions, which are entirely induced by the constant background velocity. The nonlinear fluctuation-dissipation relations for three-point functions are discussed based on holographic results.
\end{abstract}

DOI: 10.1103/PhysRevD.104.086002

\section{INTRODUCTION}

Brownian motion of a heavy particle in a thermal bath has become a textbook example [1,2] for understanding fundamental aspects of nonequilibrium statistical mechanics. The Langevin equation is perhaps the simplest effective treatment over complicated dynamics of Brownian motion:

$$
M_{\mathrm{kin}} \frac{d^{2}}{d t^{2}} q(t)+\eta \frac{d}{d t} q(t)=\xi(t)
$$

where $q(t)$ is the position of the Brownian particle, $\eta$ represents the drag force, and the stochastic variable $\xi(t)$ stands for the random force felt by the Brownian particle. Usually, the random force is assumed to be white noise:

$$
\langle\xi(t)\rangle=0, \quad\left\langle\xi(t) \xi\left(t^{\prime}\right)\right\rangle=2 T \eta \delta\left(t-t^{\prime}\right),
$$

where the strength of the noise-noise correlator is related to the drag force through the fluctuation-dissipation theorem. Here, $T$ is the temperature of the thermal bath.

The Langevin theory of Brownian motion (1.1) could be derived from the real time path integral on the closed time contour $[3,4]$ (see also [5,6]). The latter is also called Schwinger-Keldysh (SK) formalism [7-9], which provides a unified framework for describing the quantum manybody system in and out of equilibrium [9]. Within the SK

\footnotetext{
* Corresponding author. zhangbiye@hit.edu.cn

yybu@hit.edu.cn

Published by the American Physical Society under the terms of the Creative Commons Attribution 4.0 International license. Further distribution of this work must maintain attribution to the author(s) and the published article's title, journal citation, and DOI. Funded by SCOAP ${ }^{3}$.
}

formalism, the quantum system effectively evolves forward (from initial time $t_{i}$ to final time $t_{f}$ ) and then backward (from $t_{f}$ to $t_{i}$ ), forming a closed time contour, as depicted in Fig. 1. Moreover, the SK formalism systematically incorporates both fluctuations and dissipations, which is achieved by the doubling of degrees of freedom: $q \rightarrow\left(q_{1}, q_{2}\right)$, where the subscripts 1,2 denote variables on the upper and lower branches of the SK contour in Fig. 1.

Brownian motion involves two key concepts in nonequilibrium statistical mechanics: closed system versus open system [1]. Thinking of the Brownian particle and the thermal bath as a whole forms a closed system, denoted as $\{q, \Phi\}$. Dynamics of this closed system is presumably described by an action:

$$
S_{C}=S_{p}[q]+S_{\mathrm{th}}[\Phi]+S_{\mathrm{int}}[q, \Phi]
$$

where $S_{p}[q]$ is the action for the Brownian particle, $S_{\mathrm{th}}[\Phi]$ describes the microscopic theory of the constituents (collectively denoted as $\Phi$ ) for the thermal bath, and $S_{\text {int }}[q, \Phi]$ is responsible for the interactions between the Brownian particle and constituents of the thermal bath. The partition function for the closed system is

$$
Z=\int[D q][D \Phi] e^{i S_{C}}
$$

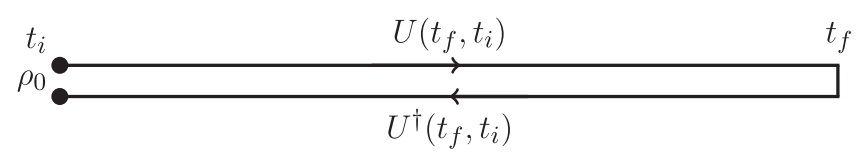

FIG. 1. The SK closed time contour: $\rho_{0}$ is the initial density matrix, and $U\left(t_{f}, t_{i}\right)$ is the time-evolution operator from initial time $t_{i}$ to final time $t_{f}$. 
However, the microscopic theory (1.3) contains too many degrees of freedom and is very hard (if not impossible) to study in general. On the other hand, the main interest is on the behavior of the Brownian particle. This motivates the concept of the open system: here, it is the Brownian particle itself, denoted as $\{q\}$. Moreover, this open system will be described by some effective theory, in which the only degree of freedom is the Brownian particle. In principle, the desired effective theory could be obtained by integrating out, in (1.4), all the degrees of freedom for the thermal bath. Then, the partition function (1.4) is equivalently expressed as a path integral over the position of the Brownian particle only,

$$
Z=\int[D q] e^{i S_{\mathrm{eff}}[q]}
$$

Certainly, in order to systematically incorporate fluctuations and dissipations, such a Wilsonian renormalization group flow should be carried out over the SK closed time contour. The resultant SK effective action $S_{\text {eff }}[q]$ will encode information of the thermal bath, interactions between the Brownian particle and the thermal bath, as well as the state.

In practice, the "integrating out" procedure outlined above is very challenging to carry out. ${ }^{1}$ It is thus natural to turn to the effective field theory (EFT) approach and construct the effective action based on the symmetry principle. In recent years, in order to tackle with dissipation, the SK formalism has been applied to formulate an EFT for dissipative hydrodynamics [12-25] (see [26] for a pedagogical review). This significant achievement for hydrodynamic EFT has largely been inspired by the AdS-CFT correspondence [27-29], which provides a tractable framework for studying dynamics of strongly coupled large $N_{c}$ (the number of colors) gauge theory via weakly coupled gravitational theory in asymptotic anti-de Sitter (AdS) space.

The Brownian motion of a heavy quark became an important example in revealing the structure of thermal noise in AdS-CFT. A heavy quark in AdS-CFT [30-34] is represented as an open string stretching from the horizon up to a probe brane, and the string's end point on the probe brane is the quark's position. ${ }^{2}$ The stochastic Langevin equation (1.1) was derived in [5] (see also [38]) via the Krustal extension [39] of finite temperature AdS-CFT [40],

\footnotetext{
${ }^{1}$ In simple examples, the degrees of freedom for the thermal bath could be integrated out, giving the linear Langevin equation [3] and its nonlinear extensions [10,11]. In these examples, microscopic details of the thermal bath are simplified as a collection of harmonic oscillators. Moreover, the interaction between the Brownian particle and bath oscillator was assumed to be either Gaussian [3] or weakly cubic [10,11].

${ }^{2}$ Interestingly, the quark string model was recently taken to understand chaos and realize the holographic dual of Sachdev-Ye-Kitaev model [35-37].
}

in which the fluctuation-dissipation theorem turns out to be an outcome of the correspondence, rather than being imposed by hand [40]. This study was further extended in a number of aspects: the heavy quark could be relativistic $[41,42]$, the environment would be in a nonequilibrium state corresponding to the time-dependent AdS black hole [43], the dissipation (the noise) would be nonlinear (non-Gaussian) [44], etc. Particularly, the latter adopted the holographic SK contour [45] (see [46-48] for an alternative holographic prescription for the SK contour) and derived the effective action for a nonlinear Langevin theory $[10,11]$. Over the past two years, the holographic SK contour [45] attracted a lot of attention in the study of SK field theory $[44,49-56]$.

In this work, we will employ the proposal [45] and explore leading order nonlinear corrections to relativistic Langevin theory of $[41,42]$. A heavy quark in a steady state motion (i.e., with a constant background velocity) corresponds to a trailing string solution in the target AdS black brane $[30,42]$. The trailing string solution induces an event horizon on the world sheet, which is different from that of target space. As clarified in $[41,42]$, it is the Hawking radiation behind this world sheet horizon that causes fluctuations on the heavy quark. Thus, the emergent AdS black brane on the world sheet will be doubled in the spirit of [45]. Perturbing around the trailing string background, we expand the string's Nambu-Goto action in the amplitude of the string perturbation: besides quadratic terms, the action also contains cubic interactions. The cubic terms are entirely induced by the quark's background velocity and are not present in [44]. Intriguingly, these cubic terms explicitly break world sheet time-reversal $(\mathcal{T})$ symmetry. The cubic interactions generate nonlinear corrections to the relativistic Langevin theory of [41,42], which do not satisfy Kubo-Martin-Schwinger (KMS) conditions for three-point functions of medium force [17,57]. This supports the claim that the fluctuations and dissipations caused by the world sheet horizon shall be of nonthermal nature [41].

The rest of this paper will be structured as follows. In Sec. II, we present the relativistic trailing string in $\mathrm{AdS}_{5}$ black brane, which is the holographic dual of a relativistic heavy quark in $\mathcal{N}=4 \mathrm{SYM}$ plasma. We also analyze nonlinear dynamics for classical string perturbation around the trailing string background. In Sec. III, we analyze the solution for classical string perturbation on the holographic SK contour associated with the string world sheet horizon. In Sec. IV, we compute the SK effective action for the relativistic heavy quark, up to cubic order in the quark's position. At quadratic order, we calculate the SK effective action first analytically in the hydrodynamic limit and then numerically for an arbitrary value of frequency. At cubic order, we compute the SK effective action analytically in a small frequency regime. In Sec. V, we make a summary of this work and 
outlook future directions. Appendixes A, B, and C supplement further calculational details.

\section{HOLOGRAPHIC SETUP}

\section{A. General statement}

The thermal bath is holographically described by Schwarzschild- $\mathrm{AdS}_{5}$ black brane (the AdS radius has been set to unity):

$$
\begin{aligned}
d s_{\mathrm{TG}}^{2} & =g_{M N} d X^{M} d X^{N} \\
& =\frac{1}{z^{2}}\left[\frac{d z^{2}}{f(z)}-f(z) d t^{2}+\delta_{i j} d X^{i} d X^{j}\right], \quad i, j=1,2,3,
\end{aligned}
$$

where $f(z)=1-z^{4} / z_{h}^{4}$. The Hawking temperature is $T=1 /\left(\pi z_{h}\right)$, which is also the temperature of the thermal bath. According to AdS/CFT correspondence, dynamics of a heavy quark is determined by an open string moving in the target space (2.1); see [30-32]. The open string stretches from the event horizon $z=z_{h}$ up to a probe brane placed near the AdS boundary, with the string's end point on the probe brane identified with the position of the heavy quark. In this work, we will focus on an infinitely heavy quark, which corresponds to placing the probe brane at the AdS boundary $z=0$.

Dynamics of an open string is described by the NambuGoto action

$$
S_{\mathrm{NG}}=-\frac{1}{2 \pi \alpha^{\prime}} \int d^{2} \sigma \sqrt{-h(X)}
$$

where $h$ is the determinant of the induced metric $h_{a b}$ on the string world sheet:

$$
d s_{\mathrm{WS}}^{2}=h_{a b} d \sigma^{a} \sigma^{b}=g_{M N} \frac{\partial X^{M}}{\partial \sigma^{a}} \frac{\partial X^{N}}{\partial \sigma^{b}} .
$$

In the static gauge, the world sheet coordinate is $\sigma^{a} \equiv(\sigma, \tau)=(z, t)$, and embedding the open string in the target space (2.1) is specified by the spatial coordinates $X^{i}\left(\sigma^{a}\right)$.

In the presence of an external Maxwell field, the NambuGoto action (2.2) should be supplemented by a boundary term:

$$
S_{\mathrm{bdy}}=\left.\int d \tau A_{M}(X) \frac{d X^{M}}{d \tau}\right|_{\mathrm{bdy}}=-\left.\int d \tau X^{M} \partial_{\tau} A_{M}(X)\right|_{\mathrm{bdy}},
$$

where in the second equality we ignored a total timederivative term.
The partition function for the bulk theory is

$$
Z_{\text {bulk }}=\int[D X]\left[D g_{M N}\right] e^{i S_{\text {bulk }}\left[X, g_{M N}\right]},
$$

where $S_{\text {bulk }}$ is the total action for the bulk theory. In the probe limit, the target space (2.1) does not fluctuate. Then, the bulk partition function $Z_{\text {bulk }}$ gets reduced to that of an open string in the curved background spacetime (2.1):

$$
Z_{\text {bulk }} \simeq Z_{\text {string }}=\int[D X] e^{i S[X]},
$$

where $S$ is the total string action

$$
S=S_{\mathrm{NG}}+S_{\mathrm{bdy}}
$$

It will be clear that the string embedding profile $X$ is a functional of the quark's position $q$, i.e., $X=X[q]$. Thus, the bulk path integral (2.6) will eventually be cast into a path integral over the position $q$. We will work in the saddle point approximation:

$$
Z_{\text {string }}=\int[D q] e^{i S[X[q]]},
$$

where $S[X[q]]$ is the on-shell classical string action. The AdS-CFT conjectures that $Z$ of (1.4) is equivalent to $Z_{\text {bulk }}$. Thus, in the probe limit, the on-shell string action $S[X[q]]$ will be identified with the effective action $S_{\text {eff }}[q]$ for the Brownian particle in a plasma medium. Therefore, the derivation of $S_{\text {eff }}[q]$ boils down to solving the classical equation of motion (EOM) for an open string in Schwarzschild $\mathrm{AdS}_{5}$.

\section{B. Trailing string in Schwarzschild-AdS $\mathrm{S}_{5}$ and holographic SK contour}

We are interested in the steady state motion of a heavy quark in $\mathcal{N}=4 \mathrm{SYM}$ plasma. On the gravity side, this corresponds to a trailing string in Schwarzschild-AdS $\mathrm{S}_{5}$ $[30,32]$. Consider a "steady" profile for the string embedding in the target space:

$$
\bar{X}^{1}=\beta t+\beta \zeta(z), \quad \bar{X}^{2}=\bar{X}^{3}=0,
$$

where we use "bar" to denote the steady state configuration. The trailing string solution was presented in $[30,32]$

$$
\zeta(z)=\frac{1}{2 \pi T}\left[\tan ^{-1}(z \pi T)-\tanh ^{-1}(z \pi T)\right] .
$$

For the configuration (2.10), the energy and momentum will flow down the string into the horizon of target space. Therefore, such a trailing string solution has to be maintained by a constant external electric field [30]. The relation between the velocity $\beta$ and electric field $E$ is determined by 


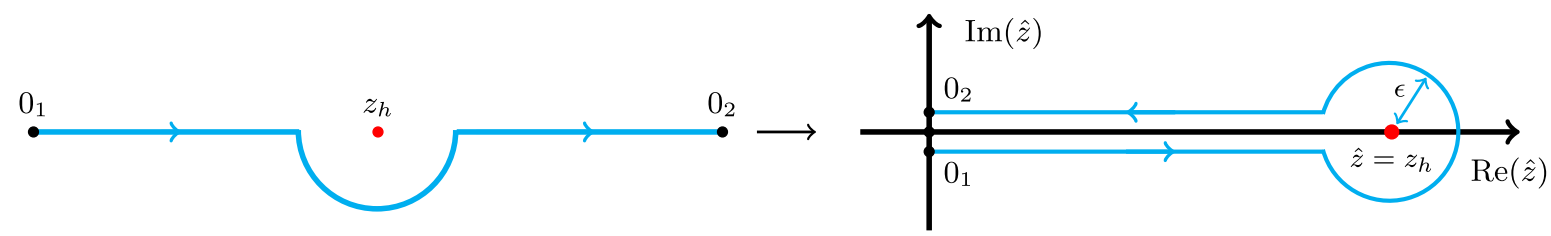

FIG. 2. From complexified (analytically continued near horizon) double AdS (left) [58] to the holographic SK contour (right) [45]. Indeed, the two horizontal legs overlap with the real axis.

the demand that the string's action is time independent and real [42]:

$\beta=\frac{2 E \alpha^{\prime}}{\sqrt{\pi^{2} T^{4}+4 E^{2} \alpha^{\prime 2}}}=\frac{2 E}{\sqrt{\lambda_{\mathrm{tH}}} \pi T^{2}} \frac{1}{\sqrt{1+\frac{4 E^{2}}{\lambda_{\mathrm{tH}} \pi^{2} T^{4}}}}$,

where $\lambda_{\mathrm{tH}}=1 / \alpha^{\prime 2}$ is the 't Hooft coupling constant [27].

Plugging the trailing string solution (2.9) and (2.10) into (2.1), we obtain an induced metric on the world sheet:

$$
\begin{aligned}
d \bar{s}_{\mathrm{WS}}^{2}= & \frac{1}{z^{2}} \frac{f(z)+\beta^{2} z^{4} / z_{h}^{4}}{f^{2}(z)} d z^{2}-\frac{2 \beta^{2}}{z_{h}^{2} f(z)} d z d t \\
& -\frac{1}{z^{2}}\left[f(z)-\beta^{2}\right] d t^{2} .
\end{aligned}
$$

Apparently, an event horizon is developed on the world sheet at $z=z_{h}\left(1-\beta^{2}\right)^{1 / 4}$, which is different from the event horizon of target space (2.1) once $\beta \neq 0$. In order to diagonalize the world sheet metric (2.12), we turn to the "hatted" coordinate system [42]:

$$
\begin{aligned}
\hat{t}= & \frac{t+\zeta_{1}(z)}{\sqrt{\gamma}}, \quad \hat{z}=\sqrt{\gamma} z, \quad \hat{X}^{i}=\sqrt{\gamma} X^{i}, \\
\zeta_{1}(z) \equiv & \frac{1}{2 \pi T}\left[\tan ^{-1}(z \pi T)-\tanh ^{-1}(z \pi T)\right] \\
& -\frac{\sqrt{\gamma}}{2 \pi T}\left[\tan ^{-1}(z \sqrt{\gamma} \pi T)-\frac{1}{2} \log \frac{1+z \sqrt{\gamma} \pi T}{1-z \sqrt{\gamma} \pi T}\right],
\end{aligned}
$$

which brings the world sheet metric (2.12) to AdS type

$$
d \bar{s}_{\mathrm{WS}}^{2}=\frac{1}{\hat{z}^{2}}\left[\frac{d \hat{z}^{2}}{f(\hat{z})}-f(\hat{z}) d \hat{t}^{2}\right]
$$

Here, the Lorentz boost factor $\gamma=1 / \sqrt{1-\beta^{2}}$.

In the ingoing EF coordinate system $\sigma^{a}=(\hat{z}, \hat{v})$ with $d \hat{v}=d \hat{t}-d \hat{z} / f(\hat{z})$, the world sheet metric is free of coordinate singularity:

$$
d \bar{s}_{\mathrm{WS}}^{2}=\frac{1}{\hat{z}^{2}}\left[-2 d \hat{v} d \hat{z}-f(\hat{z}) d \hat{v}^{2}\right] .
$$

We will be interested in the fluctuations caused by Hawking radiation emitted from the world sheet event horizon. Under the philosophy of [45], we double the world sheet
AdS and analytically continue it around the emergent event horizon $\hat{z}=z_{h}$. Resultantly, the radial coordinate $\hat{z}$ varies along the contour of Fig. 2. This holographic contour forms an ideal prescription for studying effective action for a nonequilibrium problem. The relationship between $\hat{t}$ and $\hat{v}$ is summarized below,

$$
\begin{array}{ll}
\hat{v}=\hat{t}+\chi_{2}(\hat{z}), \quad \chi_{2}(\hat{z}) \equiv-\int_{0_{2}}^{\hat{z}} \frac{d y}{f(y)}, & \hat{z} \in\left[0_{2}, z_{h}+\epsilon\right], \\
\hat{v}=\hat{t}+\chi_{1}(\hat{z}), \quad \chi_{1}(\hat{z}) \equiv-\int_{0_{1}}^{\hat{z}} \frac{d y}{f(y)}, & \hat{z} \in\left[0_{1}, z_{h}+\epsilon\right],
\end{array}
$$

where the integration constants are fixed so that $\hat{v}$ and $\hat{t}$ are equal on both AdS boundaries.

\section{String perturbation}

In order to study the stochastic motion of the heavy quark, we turn on string perturbation on top of the trailing string background reviewed in Sec. II B. Without loss of generality, we turn on string perturbation in the following way:

$\hat{X}^{1}(\hat{z}, \hat{v})=\sqrt{\gamma} \beta\left[\sqrt{\gamma} \hat{t}(\hat{v})-\zeta_{1}(\hat{z})+\zeta(\hat{z})\right]+\hat{X}_{L}(\hat{z}, \hat{v})$,

$\hat{X}^{2}(\hat{z}, \hat{v})=0+\hat{X}_{T}(\hat{z}, \hat{v}), \quad \hat{X}^{3}(\hat{z}, \hat{v})=0$,

where we only need the relation $\hat{t}(\hat{v})$ in its differential form. Here, the string perturbations $\hat{X}_{L}$ and $\hat{X}_{T}$ are small in comparison with their backgrounds, so that the Nambu-Goto action (2.2) could be expanded in powers of $\hat{X}_{L}$ and $\hat{X}_{T}$ :

$$
\begin{aligned}
S_{\mathrm{NG}} & =-\frac{1}{2 \pi \alpha^{\prime}} \int d \hat{v} \int_{0_{1}}^{0_{2}} d \hat{z} \sqrt{-\hat{h}(\hat{X})} \\
& =S_{\mathrm{NG}}^{(0)}+S_{\mathrm{NG}}^{(1)}+S_{\mathrm{NG}}^{(2)}+S_{\mathrm{NG}}^{(3)}+\cdots,
\end{aligned}
$$

where the radial integral is along the $\hat{z}$ contour of Fig. 2 in a counterclockwise way. Note that $\hat{X}^{i}(\hat{z} \rightarrow 0) / \sqrt{\gamma}$ is the position of the string end point. This shall be reflected in the AdS boundary conditions for $X_{T}, X_{L}$ to be presented later.

The zeroth order action vanishes since the integrand does not have any singularity inside the radial contour: 


$$
S_{\mathrm{NG}}^{(0)}=-\frac{1}{2 \pi \alpha^{\prime}} \int d \hat{v} \int_{0_{1}}^{0_{2}} \frac{d \hat{z}}{\hat{z}^{2}}=0
$$

The next two terms in the expansion (2.18), say $S_{\mathrm{NG}}^{(1)}$ and $S_{\mathrm{NG}}^{(2)}$, were previously discussed in [42] in the $(\hat{z}, \hat{t})$-coordinate system. Here, for completeness we record them in the hatted ingoing EF coordinate system. The first order action $S_{\mathrm{NG}}^{(1)}$ is actually a boundary term:

$$
\begin{aligned}
S_{\mathrm{NG}}^{(1)} & =\frac{1}{2 \pi \alpha^{\prime}} \int d \hat{v} \int_{0_{1}}^{0_{2}} d \hat{z} \beta \gamma(\pi T)^{2} \partial_{\hat{z}} \hat{X}_{L} \\
& =\int d \hat{v} \int_{0_{1}}^{0_{2}} d \hat{z} E \partial_{\hat{z}} \hat{X}_{L}=\left.\int d \hat{v} E \hat{X}_{L}\right|_{0_{1}} ^{0_{2}}
\end{aligned}
$$

which is exactly canceled by the first order counterpart of boundary action $S_{\text {bdy }}$ (2.4). Here, we made use of the relation (2.11).

The quadratic order action $S_{\mathrm{NG}}^{(2)}$ is

$$
\begin{aligned}
S_{\mathrm{NG}}^{(2)}= & -\frac{1}{2 \pi \alpha^{\prime}} \int d \hat{v} \int_{0_{1}}^{0_{2}} d \hat{z} \frac{1}{\hat{z}^{2}}\left[-\gamma^{2} \partial_{\hat{v}} \hat{X}_{L} \partial_{\hat{z}} \hat{X}_{L}\right. \\
& \left.+\frac{\gamma^{2}}{2} f(\hat{z})\left(\partial_{\hat{z}} \hat{X}_{L}\right)^{2}-\partial_{\hat{v}} \hat{X}_{T} \partial_{\hat{z}} \hat{X}_{T}+\frac{1}{2} f(\hat{z})\left(\partial_{\hat{z}} \hat{X}_{T}\right)^{2}\right] .
\end{aligned}
$$

Apparently, the transverse dynamics behaves the same as the static case [5], while the longitudinal dynamics gets contracted by the Lorentz boost factor $\gamma$.

The third order action $S_{\mathrm{NG}}^{(3)}$ will be the focus of the present work,

$$
\begin{aligned}
S_{\mathrm{NG}}^{(3)}= & -\frac{\beta \gamma}{2 \pi \alpha^{\prime}} \int d \hat{v} \int_{0_{1}}^{0_{2}} d \hat{z}\left\{\frac{\gamma^{2} \pi^{2} T^{2}}{2} f(\hat{z})\left(\partial_{\hat{z}} \hat{X}_{L}\right)^{3}+\frac{\pi^{2} T^{2}}{2} f(\hat{z}) \partial_{\hat{z}} \hat{X}_{L}\left(\partial_{\hat{z}} \hat{X}_{T}\right)^{2}-\frac{\gamma^{2}}{2 \hat{z}^{2}}\left(-1+3 \pi^{2} T^{2} \hat{z}^{2}\right)\left(\partial_{\hat{z}} \hat{X}_{L}\right)^{2} \partial_{\hat{v}} \hat{X}_{L}\right. \\
& -\frac{1}{2 \hat{z}^{2}}\left(1+\pi^{2} T^{2} \hat{z}^{2}\right)\left(\partial_{\hat{z}} \hat{X}_{T}\right)^{2} \partial_{\hat{v}} \hat{X}_{L}+\frac{f(\hat{z})}{\hat{z}^{2}\left(1+\pi^{2} T^{2} \hat{z}^{2}\right)} \partial_{\hat{z}} \hat{X}_{T} \partial_{\hat{z}} \hat{X}_{L} \partial_{\hat{v}} \hat{X}_{T}-\frac{\gamma^{2}}{\hat{z}^{2}\left[1+(\hat{z} \pi T)^{2}\right]} \partial_{\hat{z}} \hat{X}_{L}\left(\partial_{\hat{v}} \hat{X}_{L}\right)^{2} \\
& \left.-\frac{1}{\hat{z}^{2}\left(1+\pi^{2} T^{2} \hat{z}^{2}\right)} \partial_{\hat{z}} \hat{X}_{L}\left(\partial_{\hat{v}} \hat{X}_{T}\right)^{2}\right\} .
\end{aligned}
$$

In order to analyze time-reversal symmetry for the bulk theory, we turn to the Schwarzschild coordinate system $\sigma^{a}=(\hat{z}, \hat{t})$ momentarily, in which the NambuGoto action $S_{\mathrm{NG}}^{(2)}$ and $S_{\mathrm{NG}}^{(3)}$ are summarized in Appendix A. From the world sheet perspective, the second order action $S_{\mathrm{NG}}^{(2)}$ is invariant under the $\mathcal{T}$ transformation: $\hat{t} \rightarrow-\hat{t}, \hat{X}_{L, T}(\hat{t}, \hat{z}) \rightarrow \hat{X}_{L, T}(-\hat{t}, \hat{z})$. However, the cubic order action $S_{\mathrm{NG}}^{(3)}$ explicitly breaks $\mathcal{T}$ symmetry. $\mathcal{T}$ symmetry is crucial in deriving KMS constraints on the SK effective action [17], which will be discussed in Sec. IV.

The EOMs for $\hat{X}_{T, L}$, derived from (2.21) and (2.22), are

$$
\begin{aligned}
& 0=\partial_{\hat{z}}\left[\frac{1}{\hat{z}^{2}} f(\hat{z}) \partial_{\hat{z}} \hat{X}_{T}\right]-\frac{2}{\hat{z}^{2}} \partial_{\hat{v}} \partial_{\hat{z}} \hat{X}_{T}+\frac{2}{\hat{z}^{3}} \partial_{\hat{v}} \hat{X}_{T}+f_{T}\left[\hat{X}_{T}, \hat{X}_{L}\right], \\
& 0=\partial_{\hat{z}}\left[\frac{1}{\hat{z}^{2}} f(\hat{z}) \partial_{\hat{z}} \hat{X}_{L}\right]-\frac{2}{\hat{z}^{2}} \partial_{\hat{v}} \partial_{\hat{z}} \hat{X}_{L}+\frac{2}{\hat{z}^{3}} \partial_{\hat{v}} \hat{X}_{L}+f_{L}\left[\hat{X}_{T}, \hat{X}_{L}\right],
\end{aligned}
$$

where the nonlinear terms $f_{T}, f_{L}$ are quadratic in $\hat{X}_{T}, \hat{X}_{L}$. We do not record explicit expressions for $f_{T}, f_{L}$ since they are irrelevant for the computation of cubic order SK effective action. We will elaborate on this point later. The nonlinear EOMs (2.23) will be solved perturbatively

$$
\hat{X}_{T, L}=\alpha^{1} \hat{X}_{T, L}^{(1)}+\alpha^{2} \hat{X}_{T, L}^{(2)}+\cdots
$$

Then, at each order in the perturbative expansion (2.24), $\hat{X}_{T, L}^{(n)}$ obeys linear differential equations. Particularly, at the lowest order $\mathcal{O}\left(\alpha^{1}\right), \hat{X}_{T, L}$ satisfy the same homogeneous equation,

$$
\begin{gathered}
\partial_{\hat{z}}\left[\frac{1}{\hat{z}^{2}} f(\hat{z}) \partial_{\hat{z}} \Phi\right]-\frac{2}{\hat{z}^{2}} \partial_{\hat{v}} \partial_{\hat{z}} \Phi+\frac{2}{\hat{z}^{3}} \partial_{\hat{v}} \Phi=0, \\
\Phi=\hat{X}_{T}^{(1)} \text { or } \hat{X}_{L}^{(1)},
\end{gathered}
$$

which is analogous to EOM for a free scalar in Schwarzschild-AdS $\mathrm{A}_{2}$. The AdS boundary conditions are

$\hat{X}_{T}^{(1)}\left(\hat{z} \rightarrow 0_{s}\right)=\hat{q}_{s}^{T}, \quad \hat{X}_{T}^{(n>1)}\left(\hat{z} \rightarrow 0_{s}\right)=0, \quad s=1,2$,

$\hat{X}_{L}^{(1)}\left(\hat{z} \rightarrow 0_{s}\right)=\hat{q}_{s}^{L}, \quad \hat{X}_{L}^{(n>1)}\left(\hat{z} \rightarrow 0_{s}\right)=0, \quad s=1,2$.

Substituting the expansion (2.24) into (2.21), the quadratic order action $S_{\mathrm{NG}}^{(2)}$ can be split into two parts:

$$
S_{\mathrm{NG}}^{(2)}=S_{\mathrm{NG}}^{(2), \mathrm{P} 1}+S_{\mathrm{NG}}^{(2), \mathrm{P} 2},
$$

where 


$$
\begin{aligned}
S_{\mathrm{NG}}^{(2), \mathrm{P} 1}=-\frac{1}{2 \pi \alpha^{\prime}} \int d \hat{v} & \int_{0_{1}}^{0_{2}} d \hat{z} \frac{1}{\hat{z}^{2}}\left[-\gamma^{2} \partial_{\hat{v}} \hat{X}_{L}^{(1)} \partial_{\hat{z}} \hat{X}_{L}^{(1)}+\frac{\gamma^{2}}{2} f(\hat{z})\left(\partial_{\hat{z}} \hat{X}_{L}^{(1)}\right)^{2}-\partial_{\hat{v}} \hat{X}_{T}^{(1)} \partial_{\hat{z}} \hat{X}_{T}^{(1)}+\frac{1}{2} f(\hat{z})\left(\partial_{\hat{z}} \hat{X}_{T}^{(1)}\right)^{2}\right], \\
S_{\mathrm{NG}}^{(2), \mathrm{P} 2}= & -\frac{1}{2 \pi \alpha^{\prime}} \int d \hat{v} \int_{0_{1}}^{0_{2}} d \hat{z} \frac{1}{\hat{z}^{2}}\left[-\partial_{\hat{v}} X_{T}^{(2)} \partial_{\hat{z}} \hat{X}_{T}^{(1)}-\partial_{\hat{v}} \hat{X}_{T}^{(1)} \partial_{\hat{z}} \hat{X}_{T}^{(2)}+f(\hat{z}) \partial_{\hat{z}} \hat{X}_{T}^{(2)} \partial_{\hat{z}} \hat{X}_{T}^{(1)}\right. \\
& \left.+\gamma^{2}\left(-\partial_{\hat{v}} \hat{X}_{L}^{(2)} \partial_{\hat{z}} \hat{X}_{L}^{(1)}-\partial_{\hat{v}} \hat{X}_{L}^{(1)} \partial_{\hat{z}} \hat{X}_{L}^{(2)}+f(\hat{z}) \partial_{\hat{z}} \hat{X}_{L}^{(2)} \partial_{\hat{z}} \hat{X}_{L}^{(1)}\right)\right] .
\end{aligned}
$$

In (2.27), a piece of order $\mathcal{O}\left(\alpha^{4}\right)$ is ignored since it is beyond the scope of the present work. Upon integration by parts, (2.29) is computed as

$$
\begin{aligned}
S_{\mathrm{NG}}^{(2), \mathrm{P} 2} & =-\frac{1}{2 \pi \alpha^{\prime}} \int d \hat{v} \int_{0_{1}}^{0_{2}} d \hat{z}\left\{\partial_{\hat{z}}\left(\hat{X}_{T}^{(2)} \frac{f(\hat{z})}{\hat{z}^{2}} \partial_{\hat{z}} \hat{X}_{T}^{(1)}-\hat{X}_{T}^{(2)} \frac{1}{\hat{z}^{2}} \partial_{\hat{v}} \hat{X}_{T}^{(1)}\right)+\gamma^{2} \partial_{\hat{z}}\left(\hat{X}_{L}^{(2)} \frac{f(\hat{z})}{\hat{z}^{2}} \partial_{\hat{z}} \hat{X}_{L}^{(1)}-\hat{X}_{L}^{(2)} \frac{1}{\hat{z}^{2}} \partial_{\hat{v}} \hat{X}_{L}^{(1)}\right)\right\} \\
& =-\left.\frac{1}{2 \pi \alpha^{\prime}} \int d \hat{v}\left\{\hat{X}_{T}^{(2)}\left(\frac{f(\hat{z})}{\hat{z}^{2}} \partial_{\hat{z}} \hat{X}_{T}^{(1)}-\frac{1}{\hat{z}^{2}} \partial_{\hat{v}} \hat{X}_{T}^{(1)}\right)+\gamma^{2} \hat{X}_{L}^{(2)}\left(\frac{f(\hat{z})}{\hat{z}^{2}} \partial_{\hat{z}} \hat{X}_{L}^{(1)}-\frac{1}{\hat{z}^{2}} \partial_{\hat{v}} \hat{X}_{L}^{(1)}\right)\right\}\right|_{\hat{z}=0_{1}} ^{\hat{z}=0_{2}} \\
& =0,
\end{aligned}
$$

where the Dirichlet conditions for $\hat{X}_{T, L}^{(2)}$ at the AdS boundaries have been utilized; cf. (2.26). Moreover, in obtaining (2.30), total time derivative terms are ignored. In the same way, (2.28) is cast into a surface term:

$$
S_{\mathrm{NG}}^{(2), \mathrm{P} 1}=-\left.\frac{1}{2 \pi \alpha^{\prime}} \int d \hat{v}\left[\frac{f(\hat{z})}{2 \hat{z}^{2}} \hat{X}_{T}^{(1)} \partial_{\hat{z}} \hat{X}_{T}^{(1)}+\gamma^{2} \frac{f(\hat{z})}{2 \hat{z}^{2}} \hat{X}_{L}^{(1)} \partial_{\hat{z}} \hat{X}_{L}^{(1)}\right]\right|_{\hat{z}=0_{1}} ^{\hat{z}=0_{2}},
$$

where total time derivative terms are also ignored.

Therefore, accurate to cubic order $\mathcal{O}\left(\alpha^{3}\right)$, the total string action (Nambu-Goto action plus the boundary term) becomes

$$
S=S_{\mathrm{NG}}+S_{\mathrm{bdy}}=S_{\mathrm{NG}}^{(2), \mathrm{P} 1}+\left.S_{\mathrm{NG}}^{(3)}\right|_{\hat{X}_{T, L} \rightarrow \hat{X}_{T, L}^{(1)}}
$$

Thus, in order to derive boundary effective action up to cubic order in the position $\hat{q}^{T, L}$, we just need to solve the linearized EOMs (2.25) for string perturbations. This is similar to the case of a static heavy quark in a plasma medium [44]. Once the linearized EOMs (2.25) are solved, the core task will be to compute the cubic action $\left.S_{\mathrm{NG}}^{(3)}\right|_{\hat{X}_{T, L} \rightarrow \hat{X}_{T, L}^{(1)}}$, which involves a number of radial contour integrals.

\section{SOLUTION FOR STRING FLUCTUATION ON THE CONTOUR}

In this section, we solve the linearized EOMs (2.25) over the radial contour of Fig. 2. The solutions were previously obtained in [44] through heuristic analysis with the help of time-reversal symmetry. Here, we elaborate on the derivation for completeness, following the idea of [54]. To this end, we cut the contour in Fig. 2 at $\hat{z}=\hat{z}_{ \pm} \equiv \hat{z}_{h}+\epsilon$, where the subscripts "+" and "--" are used to distinguish between the upper and lower segments of the contour. Then, we will search for general solutions for (2.25) in the single copy of the contour, namely, either on the upper branch or on the lower branch of Fig. 2. Crucially, the general solution for the upper branch and that for the lower branch have to be carefully glued at the cutting point $\hat{z}=\hat{z}_{h}+\epsilon$. The correct gluing conditions at the cutting point $\hat{z}=$ $\hat{z}_{h}+\epsilon$ will be derived from the quadratic order action (2.21).

\section{A. Horizon gluing conditions}

Since the actions for $\hat{X}_{T}^{(1)}$ and $\hat{X}_{L}^{(1)}$ differ by an overall constant $\gamma^{2}$, the horizon gluing conditions for $\hat{X}_{T}^{(1)}$ and $\hat{X}_{L}^{(1)}$ shall be the same. So, we will take $\hat{X}_{T}^{(1)}$ as an example to derive the horizon gluing conditions. The action for $\hat{X}_{T}^{(1)}$ is

$$
\begin{aligned}
S_{\mathrm{NG}}^{(2), \mathrm{T}}= & -\frac{1}{2 \pi \alpha^{\prime}} \int d \hat{v} \int_{0_{1}}^{0_{2}} d \hat{z} \frac{1}{\hat{z}^{2}}\left[-\partial_{\hat{v}} \hat{X}_{T}^{(1)} \partial_{\hat{z}} \hat{X}_{T}^{(1)}\right. \\
& \left.+\frac{1}{2} f(\hat{z})\left(\partial_{\hat{z}} \hat{X}_{T}^{(1)}\right)^{2}\right] .
\end{aligned}
$$

In the spirit of [47], the gluing conditions at the cutting point can be derived by demanding the bulk action $S_{\mathrm{NG}}^{(2), \mathrm{T}}$ to be extremal with respect to the variation of horizon data $\hat{X}_{T}^{(1)}\left(\hat{z}_{h}+\epsilon, \hat{v}\right)$. The variation of (3.1) is 


$$
\begin{aligned}
\delta S_{\mathrm{NG}}^{(2), \mathrm{T}}= & -\frac{1}{2 \pi \alpha^{\prime}} \int d \hat{v}\left\{\left.\left(-\frac{1}{\hat{z}^{2}} \partial_{\hat{v}} \hat{X}_{T}^{(1)}+\frac{f(\hat{z})}{\hat{z}^{2}} \partial_{\hat{z}} \hat{X}_{T}^{(1)}\right) \delta \hat{X}_{T}^{(1)}\right|_{0_{1}} ^{\hat{z}_{-}}\right. \\
& \left.-\left.\left(-\frac{1}{\hat{z}^{2}} \partial_{\hat{v}} \hat{X}_{T}^{(1)}+\frac{f(\hat{z})}{\hat{z}^{2}} \partial_{\hat{z}} \hat{X}\right) \delta \hat{X}_{T}^{(1)}\right|_{0_{2}} ^{\hat{z}_{+}}\right\}
\end{aligned}
$$

where the linearized EOM for $\hat{X}_{T}^{(1)}$ [cf. (2.25)] is used. It is natural to assume that $\hat{X}_{T}^{(1)}$ is continuous across the cutting point:

$$
\hat{X}_{T}^{(1)}\left(\hat{z}_{+}\right)=\hat{X}_{T}^{(1)}\left(\hat{z}_{-}\right) .
$$

Then, the extremum condition at the cutting point gives rise to

$$
\begin{gathered}
\frac{\delta S_{\mathrm{NG}}^{(2), \mathrm{T}}}{\delta \hat{X}_{T}^{(1)}\left(\hat{z}_{h}+\epsilon, \hat{v}\right)}=0 \\
\left.\Rightarrow f(\hat{z}) \partial_{\hat{z}} \hat{X}_{T}^{(1)}\right|_{\hat{z}=\hat{z}_{+}}=\left.f(\hat{z}) \partial_{\hat{z}} \hat{X}_{T}^{(1)}\right|_{\hat{z}=\hat{z}_{-}} .
\end{gathered}
$$

Since $f(\hat{z})$ vanishes at the horizon, the gluing condition implies $\partial_{\hat{z}} \hat{X}_{T}^{(1)}$ may not be continuous across the horizon.

The same analysis can be made for the longitudinal mode $\hat{X}_{L}^{(1)}$, which will give the same gluing conditions as (3.3) and (3.4),

$\hat{X}_{T}^{(1)}\left(\hat{z}_{+}\right)=\hat{X}_{T}^{(1)}\left(\hat{z}_{-}\right),\left.\quad f(\hat{z}) \partial_{\hat{z}} \hat{X}_{L}^{(1)}\right|_{\hat{z}=\hat{z}_{+}}=\left.f(\hat{z}) \partial_{\hat{z}} \hat{X}_{L}^{(1)}\right|_{\hat{z}=\hat{z}_{-}}$.

Physically, the gluing conditions (3.3), (3.4), and (3.5) correspond to continuity for string's momentum. We will use them to glue the piecewise solutions to be found for the upper segment and the lower segment in the next two subsections.

\section{B. From linearly independent solutions to solution on the contour}

In this subsection, we search for linearly independent solutions for $\hat{X}_{T, L}^{(1)}$ on upper or lower segments of the contour. Then, the general solution (superposition of linearly independent solutions) will be glued at the cutting point, under the conditions (3.3), (3.4), and (3.5). Eventually, the AdS boundary conditions (2.26) will determine all the integration constants.

It is more convenient to turn to frequency domain

$$
\Phi(\hat{z}, \hat{v})=\int \frac{d \hat{\omega}}{2 \pi} e^{-i \hat{\omega} \hat{v}} \Phi(\hat{z}, \hat{\omega}),
$$

where the Fourier mode $\Phi(\hat{z}, \hat{\omega})$ satisfies a homogeneous ordinary differential equation (ODE),

$$
0=\partial_{\hat{z}}\left[\frac{f(\hat{z})}{\hat{z}^{2}} \partial_{\hat{z}} \Phi\right]+\frac{2}{\hat{z}^{2}} i \hat{\omega} \partial_{\hat{z}} \Phi-\frac{2}{\hat{z}^{3}} i \hat{\omega} \Phi .
$$

Apparently, the time-reversal symmetry is not realized simply as

$$
\hat{\omega} \rightarrow-\hat{\omega}, \quad \Phi(\hat{z}, \hat{\omega}) \rightarrow \Phi(\hat{z},-\hat{\omega}) .
$$

In order to see how the time-reversal symmetry is realized, we temporarily turn to Schwarzschild coordinate $(\hat{z}, \hat{t})$ for the world sheet space, in which the linearized string fluctuations $\hat{X}_{T, L}^{(1)}(\hat{z}, \hat{t})$ will be collectively denoted as $\tilde{\Phi}(\hat{z}, \hat{t})$. In the frequency domain,

$$
\tilde{\Phi}(\hat{z}, \hat{t})=\int \frac{d \hat{\omega}}{2 \pi} e^{-i \omega \hat{t}} \tilde{\Phi}(\hat{z}, \omega),
$$

where the Fourier mode $\tilde{\Phi}(\hat{z}, \hat{\omega})$ obeys

$$
0=\partial_{\hat{z}}\left[\frac{f(\hat{z})}{\hat{z}^{2}} \partial_{\hat{z}} \tilde{\Phi}\right]+\frac{\hat{\omega}^{2}}{\hat{z}^{2} f(\hat{z})} \tilde{\Phi} .
$$

Here, time-reversal symmetry is simply realized as

$$
\hat{\omega} \rightarrow-\hat{\omega}, \quad \tilde{\Phi}(\hat{z}, \hat{\omega}) \rightarrow \tilde{\Phi}(\hat{z},-\hat{\omega}),
$$

which will be helpful for finding linearly independent solutions.

In the single copy of the contour, namely, $\hat{z} \in\left[0, z_{h}\right]$, the EOM (3.10) admits two linearly independent solutions: the ingoing mode and the outgoing mode. Near the horizon, the ingoing mode behaves as

$$
\begin{aligned}
\tilde{\Phi}^{\mathrm{ig}}\left(\hat{z} \rightarrow z_{h}, \hat{\omega}\right)= & \left(z_{h}-\hat{z}\right)^{-i \hat{\omega} /(4 \pi T)}\left[\tilde{\Phi}_{h}^{0}+\tilde{\Phi}_{h}^{1}\left(z_{h}-\hat{z}\right)\right. \\
& \left.+\tilde{\Phi}_{h}^{2}\left(z_{h}-\hat{z}\right)^{2}+\cdots\right],
\end{aligned}
$$

where $\tilde{\Phi}_{h}^{1}, \tilde{\Phi}_{h}^{2}, \ldots$, are determined in terms of $\tilde{\Phi}_{h}^{0}$ and the horizon data $\tilde{\Phi}_{h}^{0}$ are undetermined. The ingoing solution $\tilde{\Phi}^{\mathrm{ig}}$ is uniquely fixed once the horizon data $\tilde{\Phi}_{h}^{0}$ are given. Indeed, the horizon data $\tilde{\Phi}_{h}^{0}$ are in one-to-one correspondence to the boundary value $\tilde{\Phi}^{\mathrm{ig}}(\hat{z}=0, \hat{\omega})$.

Under the time-reversal symmetry (3.11), the outgoing mode is

$$
\tilde{\Phi}^{\mathrm{og}}(\hat{z}, \hat{\omega})=\tilde{\Phi}^{\mathrm{ig}}(\hat{z},-\hat{\omega}) .
$$

Now, we would like to convert the relationship (3.13) into the ingoing EF coordinate. This is achieved by realizing that $\Phi$ (also $\tilde{\Phi}$ ) is a scalar in the world sheet space, which tells that

$$
\begin{aligned}
\Phi(\hat{z}, \hat{\omega}) e^{-i \hat{\omega} \hat{v}} & =\tilde{\Phi}(\hat{z}, \hat{\omega}) e^{-i \hat{\omega} \hat{t}} \\
\Rightarrow \Phi(\hat{z}, \hat{\omega}) & =\tilde{\Phi}(\hat{z}, \hat{\omega}) e^{i \hat{\omega} \chi_{s}(\hat{z})}, \quad s=1,2
\end{aligned}
$$


where the subscript " $s$ " is to remind one of the upper or the lower segment. Here, we made use of the relation (2.16). Notice that the relationship (3.14) also holds for each linearly independent solution. So, in the ingoing EF coordinate, an analog of (3.13) is

$$
\Phi^{\mathrm{og}}(\hat{z}, \hat{\omega})=e^{2 i \hat{\omega} \chi_{s}(\hat{z})} \Phi^{\mathrm{ig}}(\hat{z},-\hat{\omega}),
$$

which could be interpreted as the nonlinear realization of time-reversal symmetry [44].

Thus, we immediately write down general solutions on the upper and lower segments:

$$
\begin{aligned}
\Phi^{\mathrm{up}}(\hat{z}, \hat{\omega})= & c^{\mathrm{up}} \Phi^{\mathrm{ig}}(\hat{z}, \hat{\omega}) & \\
& +d^{\mathrm{up}} e^{2 i \hat{\omega} \chi_{2}(\hat{z})} \Phi^{\mathrm{ig}}(\hat{z},-\hat{\omega}), & \hat{z} \in\left[0_{2}, z_{h}+\epsilon\right], \\
\Phi^{\mathrm{dw}}(\hat{z}, \hat{\omega})= & c^{\mathrm{dw}} \Phi^{\mathrm{ig}}(\hat{z}, \hat{\omega}) & \\
& +d^{\mathrm{dw}} e^{2 i \hat{\omega} \chi_{1}(\hat{z})} \Phi^{\mathrm{ig}}(\hat{z},-\hat{\omega}), & \hat{z} \in\left[0_{1}, z_{h}+\epsilon\right] .
\end{aligned}
$$

Here, since the ingoing solution $\Phi^{\mathrm{ig}}$ is regular, we do not need to distinguish between the upper and lower segments. Imposing the gluing conditions (3.3), (3.4), and (3.5) on the solutions (3.16), we obtain

$$
c^{\mathrm{up}}=c^{\mathrm{dw}}, \quad d^{\mathrm{up}}=d^{\mathrm{dw}} e^{\beta_{0} \hat{\omega}},
$$

where $\beta_{0}$ is the inverse temperature $1 / T$. So, the general solutions (3.16) become

$$
\begin{aligned}
\Phi^{\mathrm{up}}(\hat{z}, \hat{\omega})= & c \Phi^{\mathrm{ig}}(\hat{z}, \hat{\omega}) \\
& +d e^{2 i \hat{\omega} \chi_{2}(\hat{z})} \Phi^{\mathrm{ig}}(\hat{z},-\hat{\omega}), \quad \hat{z} \in\left[0_{2}, z_{h}+\epsilon\right], \\
\Phi^{\mathrm{dw}}(\hat{z}, \hat{\omega})= & c \Phi^{\mathrm{ig}}(\hat{z}, \hat{\omega}) \\
& +d e^{-\beta \hat{\omega}} e^{2 i \hat{\omega} \chi_{1}(\hat{z})} \Phi^{\mathrm{ig}}(\hat{z},-\hat{\omega}), \quad \hat{z} \in\left[0_{1}, z_{h}+\epsilon\right],
\end{aligned}
$$

where a relabeling $c^{\text {up }} \rightarrow c, d^{\text {up }} \rightarrow d$ was made. The piecewise solutions (3.18) could be put into a compact form,

$\Phi(\hat{z}, \hat{\omega})=c \Phi^{\mathrm{ig}}(\hat{z}, \hat{\omega})+d e^{2 i \hat{\omega} \chi(\hat{z})} \Phi^{\mathrm{ig}}(\hat{z},-\hat{\omega}), \quad \hat{z} \in\left[0_{1}, 0_{2}\right]$,

where the function $\chi(\hat{z})$ is defined over the entire contour,

$$
\chi(\hat{z}) \equiv-\int_{0_{2}}^{\hat{z}} \frac{d y}{f(y)}, \quad \hat{z} \in\left[0_{1}, 0_{2}\right] .
$$

The integration constants $c, d$ are fixed by the AdS boundary conditions,

$$
\begin{aligned}
c \Phi^{\mathrm{ig}(0)}(\hat{\omega})+d \Phi^{\mathrm{ig}(0)}(-\hat{\omega}) & =\hat{q}_{2}(\hat{\omega}), \quad c \Phi^{\mathrm{ig}(0)}(\hat{\omega})+d e^{-\beta_{0} \hat{\omega}} \Phi^{\mathrm{ig}(0)}(-\hat{\omega})=\hat{q}_{1}(\hat{\omega}) \\
\Rightarrow c & =\frac{1}{2} \operatorname{coth} \frac{\beta_{0} \hat{\omega}}{2} \frac{\hat{q}_{a}(\hat{\omega})}{\Phi^{\mathrm{ig}(0)}(\hat{\omega})}+\frac{\hat{q}_{r}(\hat{\omega})}{\Phi^{\mathrm{ig}(0)}(\hat{\omega})}, \quad d=-\frac{\hat{q}_{a}(\hat{\omega})}{\left(1-e^{-\beta_{0} \hat{\omega}}\right) \Phi^{\mathrm{ig}(0)}(-\hat{\omega})},
\end{aligned}
$$

where $\Phi^{\mathrm{ig}(0)}(\hat{\omega})=\Phi^{\mathrm{ig}}(\hat{z}=0, \hat{\omega})$. Here, we introduced the $(r, a)$ basis:

$$
\hat{q}_{r}=\frac{1}{2}\left(\hat{q}_{1}+\hat{q}_{2}\right), \quad \hat{q}_{a}=\hat{q}_{1}-\hat{q}_{2} .
$$

Recovering the superscripts " $T, L$ " in the position $\hat{q}_{r}, \hat{q}_{a}$, we obtain explicit solutions for linearized string perturbation $\hat{X}_{T, L}^{(1)}$ over the entire contour,

$$
\begin{aligned}
\hat{X}_{T, L}^{(1)}(\hat{z}, \hat{\omega}) & =A(\hat{z}, \hat{\omega}) \hat{q}_{r}^{T, L}(\hat{\omega})+B(\hat{z}, \hat{\omega}) \hat{q}_{a}^{T, L}(\hat{\omega}), \\
\hat{z} & \in\left[0_{1}, 0_{2}\right],
\end{aligned}
$$

where

$$
\begin{aligned}
& A(\hat{z}, \hat{\omega})=\frac{\Phi^{\mathrm{ig}}(\hat{z}, \hat{\omega})}{\Phi^{\mathrm{ig}(0)}(\hat{\omega})}, \\
& B(\hat{z}, \hat{\omega})=\frac{1}{2} \operatorname{coth} \frac{\beta_{0} \hat{\omega}}{2} \frac{\Phi^{\mathrm{ig}}(\hat{z}, \hat{\omega})}{\Phi^{\mathrm{ig}(0)}(\hat{\omega})}-\frac{e^{2 i \hat{\omega} \chi(\hat{z})}}{1-e^{-\beta_{0} \hat{\omega}}} \frac{\Phi^{\mathrm{ig}}(\hat{z},-\hat{\omega})}{\Phi^{\mathrm{ig}(0)}(-\hat{\omega})} .
\end{aligned}
$$

Thus, solving linearized string perturbation over the entire contour boils down to searching for ingoing solution $\Phi^{\mathrm{ig}}(\hat{z}, \hat{\omega})$ in a single copy of the radial contour.

\section{SCHWINGER-KELDYSH EFFECTIVE ACTION FOR BROWNIAN PARTICLE}

In this section, we plug the solution for linearized string perturbation of Sec. III into $S_{\mathrm{NG}}^{(2) \text { P1 }}$ (2.31) and $S_{\mathrm{NG}}^{(3)}(2.22)$, and evaluate the radial integral, producing the SK effective action for a heavy quark,

$$
S_{\mathrm{eff}}^{(2)}=S_{\mathrm{NG}}^{(2), \mathrm{P} 1}, \quad S_{\mathrm{eff}}^{(3)}=\left.S_{\mathrm{NG}}^{(3)}\right|_{\hat{X}_{T, L} \rightarrow \hat{X}_{T, L}^{(1)}} .
$$

\section{A. Quadratic action: Colored noise}

In order to compute the quadratic order action (2.31), we need near-boundary expansion for $e^{2 i \hat{\omega} \chi_{1,2}(\hat{z})}$ : 


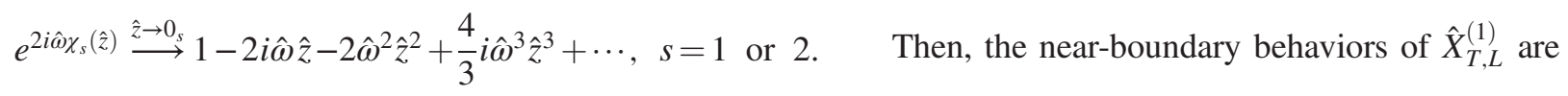

Near the AdS boundary, the ingoing mode $\Phi^{\mathrm{ig}}$ behaves as

$$
\begin{aligned}
\hat{X}_{T, L}^{(1)}\left(\hat{z} \rightarrow 0_{s}, \hat{\omega}\right) & =\hat{q}_{s}^{T, L}(\hat{\omega})-i \hat{\omega} \hat{q}_{s}^{T, L}(\hat{\omega}) \hat{z}+\mathbb{O}_{s}^{T, L}(\hat{\omega}) \hat{z}^{3}+\cdots \\
s & =1 \text { or } 2
\end{aligned}
$$

$\begin{aligned} \Phi^{\mathrm{ig}}(\hat{z} \rightarrow 0, \hat{\omega})= & \Phi^{\mathrm{ig}(0)}(\hat{\omega})-i \hat{\omega} \Phi^{\mathrm{ig}(0)}(\hat{\omega}) \hat{z}+\Phi^{\mathrm{ig}(3)}(\hat{\omega}) \hat{z}^{3} \\ & +\cdots\end{aligned}$

where the normalizable modes $\mathbb{O}_{s}^{T, L}(\hat{\omega})$ are expressed in terms of ratio $\Phi^{\mathrm{ig}(3)} / \Phi^{\mathrm{ig}(0)}$ :

$\mathbb{O}_{2}^{T, L}(\hat{\omega})=\frac{\Phi^{\mathrm{ig}(3)}(\hat{\omega})}{\Phi^{\mathrm{ig}(0)}(\hat{\omega})} \hat{q}_{r}^{T, L}(\hat{\omega})+\frac{1}{2} \operatorname{coth} \frac{\beta_{0} \hat{\omega}}{2} \frac{\Phi^{\mathrm{ig}(3)}(\hat{\omega})}{\Phi^{\mathrm{ig}(0)}(\hat{\omega})} \hat{q}_{a}^{T, L}(\hat{\omega})-\frac{1}{1-e^{-\beta_{0} \hat{\omega}}} \frac{\Phi^{\mathrm{ig}(3)}(-\hat{\omega})}{\Phi^{\mathrm{ig}(0)}(-\hat{\omega})} \hat{q}_{a}^{T, L}(\hat{\omega})+\frac{2 i \hat{\omega}^{3}}{3\left(1-e^{-\beta_{0} \hat{\omega}}\right)} \hat{q}_{a}^{T, L}(\hat{\omega})$,
$\mathbb{O}_{1}^{T, L}(\hat{\omega})=\frac{\Phi^{\mathrm{ig}(3)}(\hat{\omega})}{\Phi^{\mathrm{ig}(0)}(\hat{\omega})} \hat{q}_{r}^{T, L}(\hat{\omega})+\frac{1}{2} \operatorname{coth} \frac{\beta_{0} \hat{\omega}}{2} \frac{\Phi^{\mathrm{ig}(3)}(\hat{\omega})}{\Phi^{\mathrm{ig}(0)}(\hat{\omega})} \hat{q}_{a}^{T, L}(\hat{\omega})-\frac{e^{-\beta_{0} \hat{\omega}}}{1-e^{-\beta_{0} \hat{\omega}}} \frac{\Phi^{\mathrm{ig}(3)}(-\hat{\omega})}{\Phi^{\mathrm{ig}(0)}(-\hat{\omega})} \hat{q}_{a}^{T, L}(\hat{\omega})+\frac{2 i \hat{\omega}^{3} e^{-\beta_{0} \hat{\omega}}}{3\left(1-e^{-\beta_{0} \hat{\omega}}\right)} \hat{q}_{a}^{T, L}(\hat{\omega})$.

Plugging (4.4) into (2.31), we obtain the quadratic order SK effective action

$$
\begin{aligned}
S_{\mathrm{eff}}^{(2)}= & \frac{1}{2 \pi \alpha^{\prime}} \int \frac{d \hat{\omega}}{2 \pi}\left\{\frac{i}{2} \hat{q}_{a}^{T}(-\hat{\omega}) \hat{G}_{r r}(\hat{\omega}) \hat{q}_{a}^{T}(\hat{\omega})+\hat{q}_{a}^{T}(-\hat{\omega})\left[\hat{M} \hat{\omega}^{2}+\hat{G}_{r a}(\hat{\omega})\right] \hat{q}_{r}^{T}(\hat{\omega})\right\} \\
& +\frac{\gamma^{2}}{2 \pi \alpha^{\prime}} \int \frac{d \hat{\omega}}{2 \pi}\left\{\frac{i}{2} \hat{q}_{a}^{L}(-\hat{\omega}) \hat{G}_{r r}(\hat{\omega}) \hat{q}_{a}^{L}(\hat{\omega})+\hat{q}_{a}^{L}(-\hat{\omega})\left[\hat{M} \hat{\omega}^{2}+\hat{G}_{r a}(\hat{\omega})\right] \hat{q}_{r}^{L}(\hat{\omega})\right\},
\end{aligned}
$$

where

$$
\begin{aligned}
& \hat{G}_{r r}(\hat{\omega})=-i \operatorname{coth} \frac{\beta_{0} \hat{\omega}}{2}\left[\frac{3}{2} \frac{\Phi^{\mathrm{ig}(3)}(\hat{\omega})}{\Phi^{\mathrm{ig}(0)}(\hat{\omega})}-\frac{3}{2} \frac{\Phi^{\mathrm{ig}(3)}(-\hat{\omega})}{\Phi^{\mathrm{ig}(0)}(-\hat{\omega})}+i \hat{\omega}^{3}\right], \\
& \hat{G}_{r a}(\hat{\omega})=3 \frac{\Phi^{\mathrm{ig}(3)}(\hat{\omega})}{\Phi^{\mathrm{ig}(0)}(\hat{\omega})}+i \hat{\omega}^{3}
\end{aligned}
$$

The quadratic action (4.6) was previously obtained in [41,42] by using the Kruskal extension of the AdS/CFT correspondence [39]. In terms of the rescaled variables $\hat{q}^{T, L}$, (4.6) looks essentially the same as that of the static case [5]. In the Schwarzschild coordinate, the $i \hat{\omega}^{3}$ piece in $\hat{G}_{r r}$ and $\hat{G}_{r a}$ will be absorbed into the normalizable mode $\tilde{\Phi}^{\mathrm{ig}(3)}$ :

$$
\frac{\Phi^{\mathrm{ig}(3)}(\hat{\omega})}{\Phi^{\mathrm{ig}(0)}(\hat{\omega})}=\frac{\tilde{\Phi}^{\mathrm{ig}(3)}(\hat{\omega})}{\tilde{\Phi}^{\mathrm{ig}(0)}(\hat{\omega})}-\frac{1}{3} i \hat{\omega},
$$

which will render the correlators (4.7) to be of the same form as in [5] [see (A11) of [5]]. In (4.6), the $\hat{M} \hat{\omega}^{2}$ term arises from the divergent part of the AdS boundary limit $\hat{z} \rightarrow 0$ [5]: $\hat{M}=\lim _{\hat{z} \rightarrow 0} 1 / \hat{z}$, which is related to zerotemperature quark mass $M=\lim _{z \rightarrow 0} 1 / z$ [30] by the boost factor: $\hat{M}=M / \sqrt{\gamma}$. Apparently, the quark mass becomes finite if the quark lives on a finite cutoff slice $z=\Lambda \neq 0$.
In SK effective action, the quark position $q$ couples to plasma medium force $\mathcal{F}$ in the form $q_{1} \mathcal{F}_{1}-q_{2} \mathcal{F}_{2}=$ $q_{r} \mathcal{F}_{a}+q_{a} \mathcal{F}_{r}$ [5]. Thus, $S_{\text {eff }}$ acts as generating functional $W$ for correlators of medium force $\mathcal{F}$ :

$$
W\left[\hat{q}_{r}^{L, T}, \hat{q}_{a}^{L, T}\right]=i S_{\mathrm{eff}}\left[\hat{q}_{r}^{L, T}, \hat{q}_{a}^{L, T}\right],
$$

which, for convenience, is presented in terms of rescaled quantities. Then, we could interpret $\hat{G}_{r r}$ and $\hat{G}_{r a}$ as the symmetric and retarded force-force ${ }^{3}$ correlators [5]. Obviously, the following relation holds:

$$
\hat{G}_{r r}(\hat{\omega})=\operatorname{coth} \frac{\beta_{0} \hat{\omega}}{2} \operatorname{Im}\left[\hat{G}_{r a}(\hat{\omega})\right]
$$

which is more easily understood from the world sheet perspective. The relation (4.10) arises from the following fact: the state described by (2.15) is thermal, and the theory (2.21) preserves $\mathcal{T}$ symmetry. In terms of physical quantities by $\hat{\omega} \rightarrow \omega, \hat{q} \rightarrow q, \hat{\mathcal{F}} \rightarrow \mathcal{F},(4.10)$ will not take the exact form of the KMS condition for a thermal state, which implies the fluctuation and dissipation felt by a relativistic heavy quark is of a nonthermal nature [41].

\footnotetext{
${ }^{3}$ Here, the force should be understood as rescaled one $\hat{\mathcal{F}}=\mathcal{F} / \sqrt{\gamma}$.
} 
Via relabeling $\hat{\omega} \rightarrow-\hat{\omega}$ in the $r a$ term of (4.6), we can read off advanced correlator

$$
\hat{G}_{a r}(\hat{\omega})=3 \frac{\Phi^{\mathrm{ig}(3)}(-\hat{\omega})}{\Phi^{\mathrm{ig}(0)}(-\hat{\omega})}-i \hat{\omega}^{3},
$$

which obeys the Onsager relation $\left[\hat{G}_{r a}(\hat{\omega})\right]^{*}=\hat{G}_{a r}(\hat{\omega})$.

From (4.7), via Legendre transformation (see [5,17] for details), one can recover a stochastic equation for a heavy quark (here we set $2 \pi \alpha^{\prime}=1$ for convenience):

$$
\begin{aligned}
{\left[\hat{M} \hat{\omega}^{2}+\hat{G}_{r a}(\hat{\omega})\right] \hat{q}_{r}^{T}(\hat{\omega}) } & =\hat{\xi}^{T}(\hat{\omega}), \quad\left\langle\hat{\xi}^{T}(-\hat{\omega}) \hat{\xi}^{T}(\hat{\omega})\right\rangle=\hat{G}_{r r}(\hat{\omega}), \\
{\left[\hat{M} \hat{\omega}^{2}+\hat{G}_{r a}(\hat{\omega})\right] \hat{q}_{r}^{L}(\hat{\omega}) } & =\hat{\xi}^{L}(\hat{\omega}), \\
\left\langle\hat{\xi}^{L}(-\hat{\omega}) \hat{\xi}^{L}(\hat{\omega})\right\rangle & =\gamma^{-2} \hat{G}_{r r}(\hat{\omega}),
\end{aligned}
$$

where $\quad \hat{\xi}^{T}(\hat{\omega})=-i \hat{G}_{r r}(\hat{\omega}) \hat{q}_{a}^{T}(\hat{\omega}) \quad$ and $\quad \hat{\xi}^{L}(\hat{\omega})=$ $-i \hat{G}_{r r}(\hat{\omega}) \hat{q}_{a}^{L}(\hat{\omega})$. Here, (4.12) generalizes the Langevin equation (1.1); particularly the noise $\hat{\xi}^{T, L}$ becomes colored. It is straightforward to rewrite these equations in terms of original variables (say, $\omega, q^{T, L}, \xi^{T, L}$ ) or in a time domain; see $[41,42]$ for more details.
Below, we present our results for the retarded correlator $\hat{G}_{r a}(\hat{\omega})$ either when $\hat{\omega}$ is small (analytically) or when $\hat{\omega}$ is arbitrary (numerically).

(i) Low frequency limit

First, we consider the low frequency limit $\hat{\omega} / T \ll 1$ so that

$$
\begin{aligned}
\Phi^{\mathrm{ig}}(\hat{z}, \hat{\omega})= & \Phi_{0}^{\mathrm{ig}}(\hat{z}, \hat{\omega})+\lambda^{1} \Phi_{1}^{\mathrm{ig}}(\hat{z}, \hat{\omega}) \\
& +\lambda^{2} \Phi_{2}^{\mathrm{ig}}(\hat{z}, \hat{\omega})+\cdots,
\end{aligned}
$$

where the bookkeeping parameter $\lambda \sim \hat{\omega} / T$. Besides the regularity requirement at the horizon for $\Phi_{n}^{\mathrm{ig}}$ $(n=0,1,2, \ldots)$, we impose the AdS boundary condition,

$\Phi_{0}^{\mathrm{ig}}(\hat{z}=0, \hat{\omega})=1, \quad \Phi_{n}^{\mathrm{ig}}(\hat{z}=0, \hat{\omega})=0 \quad$ for $n \geq 1$.

In the low frequency limit $\hat{\omega} / T \ll 1$, we are able to obtain the ingoing mode $\Phi^{\mathrm{ig}}$ up to third or$\operatorname{der} \mathcal{O}\left(\hat{\omega}^{3}\right)$ :

$$
\begin{aligned}
\Phi_{0}^{\mathrm{ig}}(\hat{z}, \hat{\omega}) & =1, \\
\Phi_{1}^{\mathrm{ig}}(\hat{z}, \hat{\omega}) & =\int_{0}^{\hat{z}} \frac{x^{2} d x}{f(x)} \int_{z_{h}}^{x} \frac{2 i \hat{\omega}}{y^{3}} d y=-\frac{i \hat{\omega}}{\pi T} \arctan (\pi T z) \stackrel{\hat{z} \rightarrow 0}{\longrightarrow}-i \hat{\omega} \hat{z}+\frac{1}{3} i \hat{\omega} \pi^{2} T^{2} \hat{z}^{3}+\cdots, \\
\Phi_{2}^{\mathrm{ig}}(\hat{z}, \hat{\omega}) & =\int_{0}^{\hat{z}} \frac{x^{2} d x}{f(x)} \int_{z_{h}}^{x}\left[-\frac{2}{y^{2}} i \hat{\omega} \partial_{y} \Phi_{1}^{\mathrm{ig}}(y, \hat{\omega})+\frac{2}{y^{3}} i \hat{\omega} \Phi_{1}^{\mathrm{ig}}(y, \hat{\omega})\right] d y \\
& =-\frac{\hat{\omega}^{2}}{8 \pi T^{2}}\left[-4 \arctan (\pi T \hat{z})+4(\arctan (\pi T \hat{z}))^{2}+2 \log \frac{\left(1+\pi T \hat{z}^{2}\right)^{2}}{1+\pi^{2} T^{2} \hat{z}^{2}}\right] \stackrel{\hat{z} \rightarrow 0}{\longrightarrow}-\frac{1}{3} \hat{\omega}^{2} \pi T \hat{z}^{3}+\cdots, \\
\Phi_{3}^{\mathrm{ig}}(\hat{z}, \hat{\omega}) & =\int_{0}^{\hat{z}} \frac{x^{2} d x}{f(x)} \int_{z_{h}}^{x}\left[-\frac{2}{y^{2}} i \hat{\omega} \partial_{y} \Phi_{2}^{\mathrm{ig}}(y, \hat{\omega})+\frac{2}{y^{3}} i \hat{\omega} \Phi_{2}^{\mathrm{ig}}(y, \hat{\omega})\right] d y \stackrel{\hat{z} \rightarrow 0}{\longrightarrow} \frac{1}{12} i \hat{\omega}^{3}(-4+\pi-2 \log 2) \hat{z}^{3}+\cdots .
\end{aligned}
$$

Accordingly, the retarded two-point correlator $\hat{G}_{r a}$ is expanded as

$$
\hat{G}_{r a}(\hat{\omega})=i \hat{\omega} \pi^{2} T^{2}-\hat{\omega}^{2} \pi T+\frac{1}{4} i \hat{\omega}^{3}(\pi-2 \log 2)+\cdots,
$$

where the first two terms were reported before in [5] and the $\hat{\omega}^{3}$ term is consistent with the relevant numerical result of [44].

(ii) Beyond low frequency limit

For generic frequency $\hat{\omega}$, we numerically solve the EOM (3.7) under the regularity condition. In the ingoing EF coordinate, the analog of (3.12) is

$$
\begin{aligned}
\Phi^{\mathrm{ig}}\left(\hat{z} \rightarrow z_{h}, \hat{\omega}\right)= & \Phi_{h}^{0}+\Phi_{h}^{1}\left(z_{h}-\hat{z}\right)+\Phi_{h}^{2}\left(z_{h}-\hat{z}\right)^{2} \\
& +\Phi_{h}^{3}\left(z_{h}-\hat{z}\right)^{3}+\cdots,
\end{aligned}
$$

where $\Phi_{h}^{1}, \Phi_{h}^{2}, \Phi_{h}^{3}, \ldots$, are fixed in terms of the horizon data $\Phi_{h}^{0}$. In practical calculation, we will take $\Phi_{h}^{0}=1$ so that the near horizon expansion (4.17) provides sufficient initial conditions for the EOM (3.7). Our numerical results for $\hat{G}_{r a}(\hat{\omega})$ and $\hat{G}_{r r}(\hat{\omega})$ are shown in Fig. 3, which are in perfect agreement with [59]. While $\operatorname{Re}\left[\hat{G}_{r a}\right]$ demonstrates a damped oscillating behavior as $\hat{\omega}$ gets increased, $\operatorname{Im}\left[\hat{G}_{r a}\right]$ (also $\hat{G}_{r r}$ ) shows a cubic growing behavior at large values of $\hat{\omega}$.

\section{B. Cubic action: Non-Gaussianity}

In this subsection, we compute the cubic order effective action via replacing $\hat{X}_{T, L}$ in (2.22) by the linearized string perturbation (3.23) and then evaluating the radial integral. In the frequency domain, the cubic order action becomes 

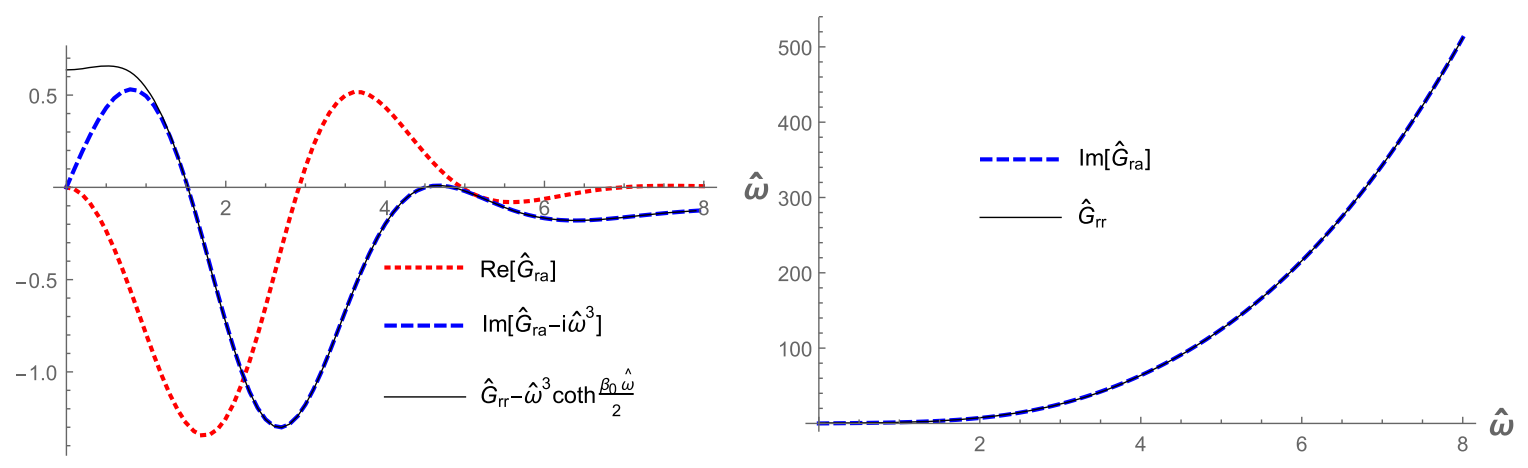

FIG. 3. Retarded and symmetric two-point correlators. Here, we set $\pi T=1$ for convenience.

$$
S_{\mathrm{NG}}^{(3)}=-\frac{\beta \gamma}{2 \pi \alpha^{\prime}} \int \frac{d \hat{\omega}_{2} d \hat{\omega}_{3}}{(2 \pi)^{2}} \delta\left(\hat{\omega}_{1}+\hat{\omega}_{2}+\hat{\omega}_{3}\right) \sum_{i=1}^{7} I_{i}\left(\hat{\omega}_{1}, \hat{\omega}_{2}, \hat{\omega}_{3}\right)
$$

where

$$
\begin{aligned}
& I_{1}=\int_{0_{1}}^{0_{2}} d \hat{z} \frac{\gamma^{2} \pi^{2} T^{2}}{2} f(\hat{z}) \partial_{\hat{z}} \hat{X}_{L}^{(1)}\left(\hat{z}, \hat{\omega}_{1}\right) \partial_{\hat{z}} \hat{X}_{L}^{(1)}\left(\hat{z}, \hat{\omega}_{2}\right) \partial_{\hat{z}} \hat{X}_{L}^{(1)}\left(\hat{z}, \hat{\omega}_{3}\right), \\
& I_{2}=\int_{0_{1}}^{0_{2}} d \hat{z} \frac{\pi^{2} T^{2}}{2} f(\hat{z}) \partial_{\hat{z}} \hat{X}_{L}^{(1)}\left(\hat{z}, \hat{\omega}_{1}\right) \partial_{\hat{z}} \hat{X}_{T}^{(1)}\left(\hat{z}, \hat{\omega}_{2}\right) \partial_{\hat{z}} \hat{X}_{T}^{(1)}\left(\hat{z}, \hat{\omega}_{3}\right), \\
& I_{3}=\int_{0_{1}}^{0_{2}} d \hat{z} \frac{-i \hat{\omega}_{3} \gamma^{2}}{2 \hat{z}^{2}}\left(1-3 \pi^{2} T^{2} \hat{z}^{2}\right) \partial_{\hat{z}} \hat{X}_{L}^{(1)}\left(\hat{z}, \hat{\omega}_{1}\right) \partial_{\hat{z}} \hat{X}_{L}^{(1)}\left(\hat{z}, \hat{\omega}_{2}\right) \hat{X}_{L}^{(1)}\left(\hat{z}, \hat{\omega}_{3}\right), \\
& I_{4}=\int_{0_{1}}^{0_{2}} d \hat{z} \frac{i \hat{\omega}_{3}}{2 \hat{z}^{2}}\left(1+\pi^{2} T^{2} \hat{z}^{2}\right) \partial_{\hat{z}} \hat{X}_{T}^{(1)}\left(\hat{z}, \hat{\omega}_{1}\right) \partial_{\hat{z}} \hat{X}_{T}^{(1)}\left(\hat{z}, \hat{\omega}_{2}\right) \hat{X}_{L}^{(1)}\left(\hat{z}, \hat{\omega}_{3}\right), \\
& I_{5}=\int_{0_{1}}^{0_{2}} d \hat{z} \frac{-i \hat{\omega}_{3} f(\hat{z})}{\hat{z}^{2}\left(1+\pi^{2} T^{2} \hat{z}^{2}\right)} \partial_{\hat{z}} \hat{X}_{T}^{(1)}\left(\hat{z}, \hat{\omega}_{1}\right) \partial_{\hat{z}} \hat{X}_{L}^{(1)}\left(\hat{z}, \hat{\omega}_{2}\right) \hat{X}_{T}^{(1)}\left(\hat{z}, \hat{\omega}_{3}\right), \\
& I_{7}=\int_{0_{1}}^{0_{2}} d \hat{z} \frac{\hat{\omega}_{2} \hat{\omega}_{3} \gamma^{2}}{\hat{z}^{2}\left(1+\pi^{2} T^{2} \hat{z}^{2}\right)} \partial_{\hat{z}} \hat{X}_{L}^{(1)}\left(\hat{z}, \hat{\omega}_{1}\right) \hat{X}_{L}^{(1)}\left(\hat{z}, \hat{\omega}_{2}\right) \hat{X}_{L}^{(1)}\left(\hat{z}, \hat{\omega}_{3}\right), \\
& 0_{0_{1}}^{0_{2}} \frac{\hat{\omega}_{2} \hat{\omega}_{3}}{\hat{z}^{2}\left(1+\pi^{2} T^{2} \hat{z}^{2}\right)} \partial_{\hat{z}} \hat{X}_{L}^{(1)}\left(\hat{z}, \hat{\omega}_{1}\right) \hat{X}_{T}^{(1)}\left(\hat{z}, \hat{\omega}_{2}\right) \hat{X}_{T}^{(1)}\left(\hat{z}, \hat{\omega}_{3}\right) .
\end{aligned}
$$

Our primary interest is to derive $S_{\text {eff }}^{(3)}$ up to first order in the time derivative, which amounts to computing (4.19) to the order $\mathcal{O}\left(\hat{\omega}^{1}\right)$. However, in order to discuss the nonlinear KMS condition, we will also track part of the $\mathcal{O}\left(\hat{\omega}^{2}\right)$ terms in $S_{\text {eff }}^{(3)}$, say arr-type terms. For a $\mathcal{P} \mathcal{T}$-invariant theory in a thermal state, the KMS condition for generating functional sets four constraints among the full set of three-point correlators [57]: they involve all $\mathcal{O}\left(\hat{\omega}^{1}\right)$ terms in the cubic action, and additional $\operatorname{arr}$ terms at the order $\mathcal{O}\left(\hat{\omega}^{2}\right)$; see Appendix $\mathrm{C}$ for a summary on KMS constraints for threepoint functions.

There is a subtle issue arising from the $\hat{\omega}$ expansion for $\hat{X}_{T, L}^{(1)}$ (3.23). While the function $A$ is regular over the contour, the function $B$ contains singularity (branch cut) near the horizon, due to the factor $e^{2 i \hat{\omega} \chi(\hat{z})}$. It is important to notice that near the horizon, the limit $\hat{\omega} \rightarrow 0$ and the limit $\epsilon \rightarrow 0$ of this factor $e^{2 i \hat{\omega} \chi(\hat{z})}$ do not commute:

$$
\lim _{\hat{\omega} \rightarrow 0} \lim _{\epsilon \rightarrow 0} e^{2 i \hat{\omega} \chi(\hat{z})} \neq \lim _{\epsilon \rightarrow 0} \lim _{\hat{\omega} \rightarrow 0} e^{2 i \hat{\omega} \chi(\hat{z})}, \quad \text { as } \hat{z} \rightarrow z_{h}+\epsilon .
$$

If we expand the factor $e^{2 i \hat{\omega} \chi(\hat{z})}$ first in the limit $\hat{\omega} \rightarrow 0$, the nature of horizon singularity for this factor will be changed. Particularly, in order to guarantee $\hat{\omega}$ expansion to be convergent, the radius $\epsilon$ of the infinitesimal circle in Fig. 2 should not be exponentially small $[5,42,45]$,

$$
1 \gg \frac{\epsilon}{z_{h}} \gg e^{-l_{\mathrm{mpp}} T},
$$

where $l_{\text {mfp }}$ is the typical timescale associated with the variations of the position $\hat{q}^{T, L}$. However, even when $l_{\mathrm{mfp}} T \rightarrow+\infty$ the condition (4.21) seems to be in tension with the limit $\epsilon \rightarrow 0$. This subtlety has been avoided in the computation of quadratic order action $S_{\mathrm{NG}}^{(2), \mathrm{P} 1}$, which is 
reduced into a boundary term without any expansion in small $\hat{\omega}$.

We have shown that subtly (4.20) is accidentally washed away in the present work. In other words, in order to extract $\mathcal{O}\left(\hat{\omega}^{1}\right)$ terms and $\mathcal{O}\left(\hat{\omega}^{2}\right)$ arr terms of (4.19), it becomes legal to expand the integrands of (4.19) in small $\hat{\omega}$ before $\epsilon \rightarrow 0$ is taken. The demonstration is technical and is deferred to Appendix B. We proceed by expanding integrands of (4.19) in the small $\hat{\omega}$ regime.

Up to $\mathcal{O}\left(\hat{\omega}^{1}\right)$, the functions $A, B(3.24)$ and their radial derivatives are expanded as

$$
\begin{aligned}
A(z, \hat{\omega}) & =1-\frac{i \hat{\omega}}{\pi T} \arctan (\pi T \hat{z})+\cdots, \\
B(\hat{z}, \hat{\omega}) & =\left[-\frac{1}{2}-\frac{2 i}{\pi} \arctan (\pi T \hat{z})-2 i T \chi(\hat{z})\right]+\hat{\omega}\left[-\frac{i}{2 \pi T} \arctan (\pi T \hat{z})-i \chi(\hat{z})+\frac{2}{\pi} \arctan (\pi T \hat{z}) \chi(\hat{z})+2 T(\chi(\hat{z}))^{2}\right]+\cdots, \\
\partial_{\hat{z}} A(\hat{z}, \hat{\omega}) & =-\frac{i \hat{\omega}}{1+\pi^{2} T^{2} \hat{z}^{2}}+\cdots, \\
\partial_{\hat{z}} B(\hat{z}, \hat{\omega}) & =\frac{2 i \pi^{2} T^{3} \hat{z}^{2}}{1-\pi^{4} T^{4} \hat{z}^{4}}+\hat{\omega}\left[\frac{i\left(1+\pi^{2} T^{2} \hat{z}^{2}\right)}{2\left(1-\pi^{4} T^{4} \hat{z}^{4}\right)}-\frac{2 \arctan (\pi T \hat{z})}{\pi\left(1-\pi^{4} T^{4} \hat{z}^{4}\right)}-\frac{2 T\left(1+\pi^{2} T^{2} \hat{z}^{2}\right)}{1-\pi^{4} T^{4} \hat{z}^{4}} \chi(\hat{z})\right]+\cdots,
\end{aligned}
$$

where $\chi(\hat{z})$ defined in (3.20) has the explicit form

$$
\chi(\hat{z})=-\frac{\arctan (\pi T \hat{z})}{2 \pi T}-\frac{\log (1+\pi T \hat{z})}{4 \pi T}+\frac{\log (1-\pi T \hat{z})}{4 \pi T}, \quad \hat{z} \in\left[0_{1}, 0_{2}\right] .
$$

Apparently, $\chi(\hat{z})$ has a branch cut near the horizon and thus is a multivalued function. In piecewise form, the function $\chi(\hat{z})$ becomes

$$
\begin{aligned}
& \chi(\hat{z})=-\frac{\arctan (\pi T \hat{z})}{2 \pi T}-\frac{\log (1+\pi T \hat{z})}{4 \pi T}+\frac{\log (1-\pi T \hat{z})}{4 \pi T}-\frac{i}{2 T}, \quad \hat{z} \in\left[0_{1}, z_{h}-\epsilon\right], \\
& \chi(\hat{z})=-\frac{\arctan (\pi T \hat{z})}{2 \pi T}-\frac{\log (1+\pi T \hat{z})}{4 \pi T}+\left.\frac{\log (1-\pi T \hat{z})}{4 \pi T}\right|_{\hat{z}=z_{h}+\epsilon e^{i \theta}}, \quad \theta \in[-\pi, \pi], \\
& \chi(\hat{z})=-\frac{\arctan (\pi T \hat{z})}{2 \pi T}-\frac{\log (1+\pi T \hat{z})}{4 \pi T}+\frac{\log (1-\pi T \hat{z})}{4 \pi T}, \quad \hat{z} \in\left[0_{2}, z_{h}-\epsilon\right] .
\end{aligned}
$$

Since the integrands of $I_{1}, I_{2}$ only involve a simple pole at $\hat{z}=z_{h}$ enclosed by the contour, they could be computed via the residue theorem. The results are

$$
\begin{aligned}
& I_{1}=\frac{3 \gamma^{2} \pi^{2} T^{3}}{2}\left[T \hat{q}_{a}^{L}\left(\hat{\omega}_{1}\right) \hat{q}_{a}^{L}\left(\hat{\omega}_{2}\right) \hat{q}_{a}^{L}\left(\hat{\omega}_{3}\right)+\hat{\omega}_{1} \hat{q}_{a}^{L}\left(\hat{\omega}_{2}\right) \hat{q}_{a}^{L}\left(\hat{\omega}_{3}\right) \hat{q}_{r}^{L}\left(\hat{\omega}_{1}\right)\right], \\
& I_{2}=\frac{3 \pi^{2} T^{3}}{2}\left[T \hat{q}_{a}^{L}\left(\hat{\omega}_{1}\right) \hat{q}_{a}^{T}\left(\hat{\omega}_{2}\right) \hat{q}_{a}^{T}\left(\hat{\omega}_{3}\right)+\hat{\omega}_{1} \hat{q}_{a}^{L}\left(\hat{\omega}_{2}\right) \hat{q}_{a}^{T}\left(\hat{\omega}_{3}\right) \hat{q}_{r}^{T}\left(\hat{\omega}_{1}\right)\right] .
\end{aligned}
$$

The integrals $I_{3,4,5}$ will involve the logarithmic branch cut at $\hat{z}=z_{h}$ and will be computed by splitting the radial contour of Fig. 2 as

$$
\int_{0_{1}}^{0_{2}} d \hat{z}=\int_{0_{1}}^{z_{h}-\epsilon} d \hat{z}+\int_{\mathcal{C}} d \theta+\int_{z_{h}-\epsilon}^{0_{2}} d \hat{z}
$$

where the integrals over the two horizontal legs will be partially canceled. The integrals $I_{6,7}$ do not contribute at the order $\mathcal{O}\left(\hat{\omega}^{1}\right)$. For illustration we take the computation of $I_{3}$ as an example. For convenience, we split $I_{3}$ into three parts,

$$
I_{3}=I_{3}^{(1)} \hat{q}_{a}^{L}\left(\hat{\omega}_{1}\right) \hat{q}_{a}^{L}\left(\hat{\omega}_{2}\right) \hat{q}_{r}^{L}\left(\hat{\omega}_{3}\right)+I_{3}^{(2)} \hat{q}_{a}^{L}\left(\hat{\omega}_{1}\right) \hat{q}_{a}^{L}\left(\hat{\omega}_{2}\right) \hat{q}_{a}^{L}\left(\hat{\omega}_{3}\right)+I_{3}^{(3)} \hat{q}_{a}^{L}\left(\hat{\omega}_{1}\right) \hat{q}_{a}^{L}\left(\hat{\omega}_{2}\right) \hat{q}_{a}^{L}\left(\hat{\omega}_{3}\right),
$$

where $I_{3}^{(1)}$ and $I_{3}^{(2)}$ are computed directly via the residue theorem, 


$$
\begin{aligned}
I_{3}^{(1)} & =\int_{0_{1}}^{0_{2}} d \hat{z} \frac{2 i \hat{\omega}_{3} \gamma^{2} \pi^{4} T^{6} \hat{z}^{2}\left(1-3 \pi^{2} T^{2} \hat{z}^{2}\right)}{\left(1-\pi^{4} T^{4} \hat{z}^{4}\right)^{2}}=\gamma^{2} \pi^{2} T^{3} \hat{\omega}_{3}, \\
I_{3}^{(2)} & =\int_{0_{1}}^{0_{2}} d \hat{z} \frac{\gamma^{2} \pi^{3} T^{6} \hat{\omega}_{3} \hat{z}^{2}\left(-1+3 \pi^{2} T^{2} \hat{z}^{2}\right)}{\left(1-\pi^{4} T^{4} \hat{z}^{4}\right)^{2}}[i \pi-2 \arctan (\pi T \hat{z})+\log (1+\pi T \hat{z})] \\
& =-\frac{1}{8} \gamma^{2} \pi T^{3} \hat{\omega}_{3}[i+(4+2 i) \pi-4 i \log 2] .
\end{aligned}
$$

The third part is

$$
I_{3}^{(3)}=\int_{0_{1}}^{0_{2}} d \hat{z} \frac{\gamma^{2} \pi^{3} T^{6} \hat{\omega}_{3} \hat{z}^{2}\left(1-3 \pi^{2} T^{2} \hat{z}^{2}\right)}{\left(1-\pi^{4} T^{4} \hat{z}^{4}\right)^{2}} \log (1-\pi T \hat{z})
$$

where the integrand involves a branch cut at $\hat{z}=z_{h}$. Thus, $I_{3}^{(3)}$ will be computed by splitting the contour as in (4.26). The phase of the argument $\hat{z}$ is chosen as in (4.24). The contribution from the horizontal legs is

$$
\begin{aligned}
& \int_{0}^{1 /(\pi T)-\epsilon} d \hat{z} \frac{\gamma^{2} \pi^{3} T^{6} \hat{\omega}_{3} \hat{z}^{2}\left(1-3 \pi^{2} T^{2} \hat{z}^{2}\right)}{\left(1-\pi^{4} T^{4} \hat{z}^{4}\right)^{2}}(-2 i \pi) \\
& \quad=\frac{i \hat{\omega}_{3} \gamma^{2} T^{2}}{4 \epsilon}+\frac{1}{2} i \hat{\omega}_{3} \gamma^{2} \pi T^{3} \log \epsilon-\frac{1}{8} i \hat{\omega}_{3} \gamma^{2} \pi T^{3}(-3+\pi+4 \log 2-4 \log (\pi T))+\cdots,
\end{aligned}
$$

where the infrared divergences will be exactly canceled by the integral over the infinitesimal circle. In order to obtain the contribution from the infinitesimal circle, we turn to polar coordinate $\hat{z}=1 /(\pi T)+\epsilon e^{i \theta}$,

$$
\begin{array}{rl}
\int_{\mathcal{C}} & d \hat{z} \frac{\gamma^{2} \pi^{3} T^{6} \hat{\omega}_{3} \hat{z}^{2}\left(1-3 \pi^{2} T^{2} \hat{z}^{2}\right)}{\left(1-\pi^{4} T^{4} \hat{z}^{4}\right)^{2}} \log (1-\pi T \hat{z}) \\
& =\left.\int_{-\pi}^{\pi} d \theta i \epsilon e^{i \theta} \frac{\gamma^{2} \pi^{3} T^{6} \hat{\omega}_{3} \hat{z}^{2}\left(1-3 \pi^{2} T^{2} \hat{z}^{2}\right)}{\left(1-\pi^{4} T^{4} \hat{z}^{4}\right)^{2}}[\log (\pi T \epsilon)+i \pi+i \theta]\right|_{\hat{z} \rightarrow 1 /(\pi T)+\epsilon e^{i \theta}} \\
& =-\frac{i \hat{\omega}_{3} \gamma^{2} T^{2}}{4 \epsilon}-\frac{i \hat{\omega}_{3} \gamma^{2} \pi T^{3}}{2} \log \epsilon+\frac{1}{2} \gamma^{2} \hat{\omega}_{3} \pi^{2} T^{3}-\frac{1}{2} i \hat{\omega}_{3} \gamma^{2} \pi T^{3} \log (\pi T)+\cdots
\end{array}
$$

So, the result for $I_{3}$ is

$$
I_{3}=\gamma^{2} \pi^{2} T^{3} \hat{\omega}_{3} \hat{q}_{a}^{L}\left(\hat{\omega}_{1}\right) \hat{q}_{a}^{L}\left(\hat{\omega}_{2}\right) \hat{q}_{r}^{L}\left(\hat{\omega}_{3}\right)-\frac{1}{8} i \hat{\omega}_{3} \gamma^{2} \pi T^{3}(3 \pi-2) \hat{q}_{a}^{L}\left(\hat{\omega}_{1}\right) \hat{q}_{a}^{L}\left(\hat{\omega}_{2}\right) \hat{q}_{a}^{L}\left(\hat{\omega}_{3}\right)
$$

The integrals $I_{4}, I_{5}$ could be computed in parallel with $I_{3}$. For brevity, we just record the final results,

$$
\begin{aligned}
I_{4}= & -\frac{1}{8} i \hat{\omega}_{3} \pi T^{3}(2+\pi) \hat{q}_{a}^{L}\left(\hat{\omega}_{3}\right) \hat{q}_{a}^{T}\left(\hat{\omega}_{1}\right) \hat{q}_{a}^{T}\left(\hat{\omega}_{2}\right), \\
I_{5}= & \pi^{2} T^{3} \hat{\omega}_{3} \hat{q}_{a}^{L}\left(\hat{\omega}_{2}\right) \hat{q}_{a}^{T}\left(\hat{\omega}_{1}\right) \hat{q}_{r}^{T}\left(\hat{\omega}_{3}\right) \\
& -\frac{1}{4} i \hat{\omega}_{3} \pi T^{3}(\pi-2) \hat{q}_{a}^{L}\left(\hat{\omega}_{2}\right) \hat{q}_{a}^{T}\left(\hat{\omega}_{1}\right) \hat{q}_{a}^{T}\left(\hat{\omega}_{3}\right) .
\end{aligned}
$$

Finally, at the order $\mathcal{O}\left(\hat{\omega}^{2}\right)$ we only consider $\operatorname{arr}$ terms in the cubic action. The integrals $I_{1}, I_{2}, I_{5}$ do not contribute. The contributions from $I_{3,4,6,7}$ are simply computed via the residue theorem. The results are

$$
\begin{aligned}
& I_{3}^{a r r}=\gamma^{2} \pi^{2} T^{2} \hat{\omega}_{1} \hat{\omega}_{3} \hat{q}_{r}^{L}\left(\hat{\omega}_{1}\right) \hat{q}_{a}^{L}\left(\hat{\omega}_{2}\right) \hat{q}_{r}^{L}\left(\hat{\omega}_{3}\right), \\
& I_{4}^{a r r}=\pi^{2} T^{2} \hat{\omega}_{1} \hat{\omega}_{3} \hat{q}_{r}^{T}\left(\hat{\omega}_{1}\right) \hat{q}_{a}^{T}\left(\hat{\omega}_{2}\right) \hat{q}_{r}^{L}\left(\hat{\omega}_{3}\right), \\
& I_{6}^{a r r}=\frac{\gamma^{2} \pi^{2} T^{2}}{2} \hat{\omega}_{2} \hat{\omega}_{3} \hat{q}_{a}^{L}\left(\hat{\omega}_{1}\right) \hat{q}_{r}^{L}\left(\hat{\omega}_{2}\right) \hat{q}_{r}^{L}\left(\hat{\omega}_{3}\right), \\
& I_{7}^{a r r}=\frac{\pi^{2} T^{2}}{2} \hat{\omega}_{2} \hat{\omega}_{3} \hat{q}_{a}^{L}\left(\hat{\omega}_{1}\right) \hat{q}_{r}^{T}\left(\hat{\omega}_{2}\right) \hat{q}_{r}^{T}\left(\hat{\omega}_{3}\right) .
\end{aligned}
$$

Combining (4.25), (4.32), (4.33), and (4.34), we obtain the cubic order SK effective action,

$$
S_{\mathrm{eff}}^{(3)}=S_{\mathrm{eff}}^{(3) L L L}+S_{\mathrm{eff}}^{(3) L T T}
$$

where 


$$
\begin{aligned}
S_{\mathrm{eff}}^{(3) L L L}= & \frac{\beta \gamma}{2 \pi \alpha^{\prime}} \int \frac{d \hat{\omega}_{2} d \hat{\omega}_{3}}{(2 \pi)^{2}}\left\{\left[-\frac{3}{2} \gamma^{2} \pi^{2} T^{4}+\frac{1}{8} \gamma^{2} \pi(3 \pi-2) T^{3} i \hat{\omega}_{3}\right] \hat{q}_{a}^{L}\left(\hat{\omega}_{1}\right) \hat{q}_{a}^{L}\left(\hat{\omega}_{2}\right) \hat{q}_{a}^{L}\left(\hat{\omega}_{3}\right)\right. \\
& \left.-\frac{5}{2} \gamma^{2} \pi^{2} T^{3} \hat{\omega}_{3} \hat{q}_{a}^{L}\left(\hat{\omega}_{1}\right) \hat{q}_{a}^{L}\left(\hat{\omega}_{2}\right) \hat{q}_{r}^{L}\left(\hat{\omega}_{3}\right)-\frac{3}{2} \gamma^{2} \pi^{2} T^{2} \hat{\omega}_{2} \hat{\omega}_{3} \hat{q}_{a}^{L}\left(\hat{\omega}_{1}\right) \hat{q}_{r}^{L}\left(\hat{\omega}_{2}\right) \hat{q}_{r}^{L}\left(\hat{\omega}_{3}\right)\right\}, \\
S_{\mathrm{eff}}^{(3) L T T}= & \frac{\beta \gamma}{2 \pi \alpha^{\prime}} \int \frac{d \hat{\omega}_{2} d \hat{\omega}_{3}}{(2 \pi)^{2}}\left\{\left[-\frac{3}{2} \pi^{2} T^{4}+\frac{1}{8} \pi(\pi+2) T^{3} i \hat{\omega}_{1}+\frac{1}{4} \pi(\pi-2) T^{3} i \hat{\omega}_{3}\right]\right. \\
& \times \hat{q}_{a}^{L}\left(\hat{\omega}_{1}\right) \hat{q}_{a}^{T}\left(\hat{\omega}_{2}\right) \hat{q}_{a}^{T}\left(\hat{\omega}_{3}\right)-\frac{5}{2} \pi^{2} T^{3} \hat{\omega}_{3} \hat{q}_{a}^{L}\left(\hat{\omega}_{1}\right) \hat{q}_{a}^{T}\left(\hat{\omega}_{2}\right) \hat{q}_{r}^{T}\left(\hat{\omega}_{3}\right)-\pi^{2} T^{2} \hat{\omega}_{1} \hat{\omega}_{3} \\
& \left.\times \hat{q}_{r}^{T}\left(\hat{\omega}_{1}\right) \hat{q}_{a}^{T}\left(\hat{\omega}_{2}\right) \hat{q}_{r}^{L}\left(\hat{\omega}_{3}\right)-\frac{1}{2} \pi^{2} T^{2} \hat{\omega}_{2} \hat{\omega}_{3} \hat{q}_{a}^{L}\left(\hat{\omega}_{1}\right) \hat{q}_{r}^{T}\left(\hat{\omega}_{2}\right) \hat{q}_{r}^{T}\left(\hat{\omega}_{3}\right)\right\},
\end{aligned}
$$

where $\hat{\omega}_{1}+\hat{\omega}_{2}+\hat{\omega}_{3}=0$ is assumed. Recall that at the order $\mathcal{O}\left(\hat{\omega}^{2}\right)$ we only track $a r r$-type terms. Equation (4.36) is the main result of this work.

The cubic order action (4.36) represents not only nonlinear interactions among noises (the $a a a$-type terms) but also nonlinear interactions between dynamical variable $\hat{q}_{r}$ and noise (the aar-type terms), which are usually not covered in stochastic formulation, such as (1.1). Moreover, the aar-type terms are analogous to multiplicative noise in classical stochastic models [2]. The arr-type terms represent nonlinear $\left(\partial_{t} q\right)^{2}$ corrections to the Langevin equation (1.1). However, the presence of aaa-type terms or aar-type terms makes it inconvenient to convert (via Legendre transformation) the effective actions (4.6) and (4.36) into a stochastic equation for a heavy quark position [17]. In fact, many problems, although initially formulated as (generalized) Langevin equations with certain stochastic forces, are indeed studied by converting into an action framework (see [2]). It is thus interesting to explore physical consequences of these terms by computing loop corrections to various observables, along the line of $[23,25,60,61]$, which goes beyond the scope of present work.

Consistency check

(i) $Z_{2}$ reflection symmetry

Under the exchange of $\hat{q}_{1}^{L, T} \leftrightarrow \hat{q}_{2}^{L, T}$, the SK effective action shall satisfy [17]

$$
\left(S_{\text {eff }}\left[\hat{q}_{1}^{T}, \hat{q}_{1}^{L} ; \hat{q}_{2}^{T}, \hat{q}_{2}^{L}\right]\right)^{*}=-S_{\text {eff }}\left[\hat{q}_{2}^{T}, \hat{q}_{2}^{L} ; \hat{q}_{1}^{T}, \hat{q}_{1}^{L}\right],
$$

which is actually a unitary requirement, representing a self-consistent condition of the SK formalism. Under the constraint (4.37), in the effective action (in the time domain), the coefficient of any term with even numbers of $a$-type fields should be purely imaginary, while the coefficient of a term with odd numbers of $a$-type fields must be purely real. Obviously, our results for quadratic and cubic actions pass this basic requirement.

(ii) KMS constraints for three-point functions

From the perspective of a string world sheet, we work around a thermal state (an emergent one on the world sheet) with a constant temperature, say (2.14) or (2.15). As analyzed in Appendix A, while the quadratic bulk action (2.21) preserves the world sheet $\mathcal{T}$ symmetry, the cubic bulk action (2.22) does explicitly break it. On the other hand, the KMS constraints (C5) become applicable only if the theory is $\mathcal{T}$ invariant [17]. Thus, it is reasonable to expect that the cubic order results (4.36) will not satisfy equilibrium KMS constraints (C5), which we examine now.

Comparing (4.36) with (C1)-(C4), we can read off three-point correlators for medium force $\hat{\mathcal{F}}$. Let us take the $L L L$-sector as an example (see Appendix $\mathrm{C}$ for nation convention):

$$
\begin{aligned}
\frac{1}{3 !} \hat{G}^{L L L} & =-\frac{3 \gamma^{2} \pi^{2} T^{4}}{2}+\frac{1}{8} i \hat{\omega}_{3} \gamma^{2} \pi T^{3}(3 \pi-2), \\
\frac{i}{2} \hat{H}_{1}^{L L L} & =-\frac{5 \gamma^{2} \pi^{2} T^{3}}{2} \hat{\omega}_{1}, \quad \frac{i}{2} \hat{H}_{2}^{L L L}=-\frac{5 \gamma^{2} \pi^{2} T^{3}}{2} \hat{\omega}_{2}, \quad \frac{i}{2} \hat{H}_{3}^{L L L}=-\frac{5 \gamma^{2} \pi^{2} T^{3}}{2} \hat{\omega}_{3}, \\
\frac{1}{2} \hat{K}_{1}^{L L L} & =-\frac{3 \gamma^{2} \pi^{2} T^{2}}{2} \hat{\omega}_{2} \hat{\omega}_{3}, \quad \frac{1}{2} \hat{K}_{2}^{L L L}=-\frac{3 \gamma^{2} \pi^{2} T^{2}}{2} \hat{\omega}_{1} \hat{\omega}_{3}, \quad \frac{1}{2} \hat{K}_{3}^{L L L}=-\frac{3 \gamma^{2} \pi^{2} T^{2}}{2} \hat{\omega}_{1} \hat{\omega}_{2},
\end{aligned}
$$


where "hatted" $\hat{G}, \hat{H}, \hat{K}$ mean correlators of rescaled medium force $\hat{\mathcal{F}}$. In (4.38), an overall factor $\beta \gamma /\left(2 \pi \alpha^{\prime}\right)$ is ignored. Apparently, our results (4.38) do not satisfy (C5). The same conclusion holds for the LTT sector.

\section{SUMMARY AND OUTLOOK}

In this work, we considered nonlinear corrections to stochastic dynamics of a relativistic heavy quark in $\mathcal{N}=4$ SYM plasma, holographically described by a trailing string in $\mathrm{AdS}_{5}$ black brane $[30,41,42]$. Based on the holographic SK contour [45], we derived the SK effective action for the heavy quark, up to cubic order in the quark's position.

At the quadratic level, the SK effective action is computed first in the small $\hat{\omega}$ limit and then for the arbitrary value of $\hat{\omega}$, perfectly satisfying the "world sheet" KMS condition [see (4.10)]. At cubic order, the SK effective action is computed perturbatively in the small $\hat{\omega}$ regime. We observed that the KMS conditions for three-point functions of medium force are no longer obeyed due to steady state motion of the heavy quark. This becomes more transparent from a string world sheet's viewpoint: while the state of a trailing string results in an emergent static AdS black hole background on the string world sheet, it also renders the cubic order bulk action to break world sheet $\mathcal{T}$ invariance (see Appendix A).

Another outcome of this work is on the technical side. We carried out a preliminary study over the subtle issue arising from the noncommutative feature of the following two limits: the low frequency limit $\hat{\omega} / T \rightarrow 0$ versus $\epsilon \rightarrow 0$. Through brute-force calculations, we demonstrated that this subtlety is accidently absent in the calculation of SK effective action up to $\mathcal{O}\left(\hat{\omega}^{1}\right)$. However, this would not hold beyond first order in the time derivative and requires further investigation. We leave this open question for future study.
The present work focused on the steady state motion of a Brownian particle in a static plasma medium. This can be extended in a few directions:

(i) First, it is interesting to consider a heavy quark moving in a viscous neutral flow background [62-65], which is dual to an open string probing the slowly varying AdS black brane [66]. Such a study would be helpful in understanding fluctuationdissipation relations for a nonequilibrium state $[43,67,68]$.

(ii) Second, the present study would be extended to more realistic scenarios, say in the context of holographic models closer to QCD plasma, as considered recently in [59,69-82].

(iii) Last but not least, it is also interesting to consider spatially extended objects, and particularly when the low energy dynamics involves densities of conserved charges. One such example is the quantum critical system driven by a strong electric field [83], aiming at revealing universal scaling behaviors for an out-of-equilibrium situation. A second interesting instance is the steady state bubble nucleated within a chiral phase transition [84]. It is worth exploring nonlinear stochastic effects in both examples via the holographic technique.

\section{ACKNOWLEDGMENTS}

This work was supported by the Natural Science Foundation of China (NSFC) under Grant No. 11705037.

\section{APPENDIX A: PERTURBATIVE $S_{\mathrm{NG}}$ IN SCHWARZSCHILD COORDINATE}

In Sec. II C, the perturbative Nambu-Goto actions (2.21) and (2.22) are presented in the ingoing EF coordinate (2.15), which makes the properties under $\mathcal{T}$ transformation obscure. In Schwarzschild coordinate (2.14), the perturbative Nambu-Goto actions (2.21) and (2.22) become

$$
\begin{aligned}
& S_{\mathrm{NG}}^{(2)}=-\frac{1}{2 \pi \alpha^{\prime}} \int d \hat{t} \int_{0_{1}}^{0_{2}} \frac{d \hat{z}}{2 \hat{z}^{2}}\left[-\frac{\gamma^{2}}{f(\hat{z})}\left(\partial_{\hat{t}} \hat{X}_{L}\right)^{2}+\gamma^{2} f(\hat{z})\left(\partial_{\hat{z}} \hat{X}_{L}\right)^{2}-\frac{1}{f(\hat{z})}\left(\partial_{\hat{t}} \hat{X}_{T}\right)^{2}+f(\hat{z})\left(\partial_{\hat{z}} \hat{X}_{T}\right)^{2}\right], \\
& S_{\mathrm{NG}}^{(3)}=-\frac{\gamma \beta}{2 \pi \alpha^{\prime}} \int d \hat{t} \int_{0_{1}}^{0_{2}} \frac{d \hat{z}}{2 \hat{z}^{2} f^{2}(\hat{z})}\left\{-\gamma^{2}\left(\partial_{\hat{t}} \hat{X}_{L}\right)^{3}-\gamma^{2}(\pi T \hat{z})^{2} f(\hat{z})\left(\partial_{\hat{t}} \hat{X}_{L}\right)^{2} \partial_{\hat{z}} \hat{X}_{L}\right. \\
&-\partial_{\hat{t}} \hat{X}_{L}\left(\partial_{\hat{t}} \hat{X}_{T}\right)^{2}-2(\pi T \hat{z})^{2} f(\hat{z}) \partial_{\hat{t}} \hat{X}_{L} \partial_{\hat{t}} \hat{X}_{T} \partial_{\hat{z}} \hat{X}_{T}+\gamma^{2} f^{2}(\hat{z}) \partial_{\hat{t}} \hat{X}_{L}\left(\partial_{\hat{z}} \hat{X}_{L}\right)^{2} \\
&-f^{2}(\hat{z}) \partial_{\hat{t}} \hat{X}_{L}\left(\partial_{\hat{z}} \hat{X}_{T}\right)^{2}+(\pi T \hat{z})^{2} f(\hat{z}) \partial_{\hat{z}} \hat{X}_{L}\left(\partial_{\hat{t}} \hat{X}_{T}\right)^{2}+2 f^{2}(\hat{z}) \partial_{\hat{z}} \hat{X}_{L} \partial_{\hat{t}} \hat{X}_{T} \partial_{\hat{z}} \hat{X}_{T} \\
&\left.+\gamma^{2}(\pi T \hat{z})^{2} f^{3}(\hat{z})\left(\partial_{\hat{z}} \hat{X}_{L}\right)^{3}+(\pi T \hat{z})^{2} f^{3}(\hat{z}) \partial_{\hat{z}} \hat{X}_{L}\left(\partial_{\hat{z}} \hat{X}_{T}\right)^{2}\right\} .
\end{aligned}
$$

Now, from the world sheet perspective, the $\mathcal{T}$ symmetry becomes more transparent. The second order action (A1) is invariant under the $\mathcal{T}$ transformation:

$$
\mathcal{T}: \hat{t} \rightarrow-\hat{t}, \quad \hat{X}_{T, L}(\hat{z}, \hat{t}) \rightarrow \hat{X}_{T, L}(\hat{z},-\hat{t})
$$


However, the cubic order action (A2) is not invariant under the $\mathcal{T}$ transformation above. Moreover, it does not make sense to split $S_{\mathrm{NG}}^{(3)}$ into a $\mathcal{T}$-invariant part and a $\mathcal{T}$-breaking part, since it is the whole object $S_{\mathrm{NG}}^{(3)}$ that is a Lorentzian scalar. In some sense, the $\mathcal{T}$-breaking action $S_{\mathrm{NG}}^{(3)}$ is a reflection of steady state motion for the quark.

\section{APPENDIX B: SUBTLETY DUE TO NONCOMMUTATIVITY OF $\epsilon \rightarrow 0$ VERSUS $\hat{\boldsymbol{\omega}} \rightarrow 0$}

In this Appendix, we demonstrate that the noncommutativity issue of $\epsilon \rightarrow 0$ versus $\hat{\omega} \rightarrow 0$ becomes accidentally absent for the present study. This validates the treatment of Sec. IV B.

Recall that each integrand in (4.19) potentially has an oscillating factor $(1-\pi T \hat{z})^{i \hat{\omega} /(2 \pi T)}$, which results in the noncommutativity issue (4.20). With the expression (4.23), the factor $e^{i \hat{\omega} \chi(\hat{z})}$ can be rewritten as a product of an oscillating part and a regular part:

$$
\begin{aligned}
e^{i \hat{\omega} \chi(\hat{z})}= & (1-\pi T \hat{z})^{i \hat{\omega} /(2 \pi T)} \\
& \times \exp \left\{-\frac{i \hat{\omega}}{\pi T} \arctan (\pi T \hat{z})-\frac{i \hat{\omega}}{2 \pi T} \log (1+\pi T \hat{z})\right\},
\end{aligned}
$$

where the singular portion $(1-\pi T \hat{z})^{i \hat{\omega} /(2 \pi T)}$ will not be expanded in the small $\hat{\omega}$ limit before $\epsilon \rightarrow 0$. Once the integrals (4.19) are done, we extract the low frequency limits.

We find it more convenient to rewrite $B$ of (3.24) as

$$
\begin{aligned}
B(\hat{z}, \hat{\omega}) & =B_{1}(\hat{z}, \hat{\omega})+B_{2}(\hat{z}, \hat{\omega}) \\
& =B_{1}(\hat{z}, \hat{\omega})+(1-\pi T \hat{z})^{i \hat{\omega} /(2 \pi T)} C(\hat{z}, \hat{\omega}),
\end{aligned}
$$

where $B_{1}, C$ are regular functions over the domain enclosed by the contour

$$
\begin{aligned}
B_{1}(\hat{z}, \hat{\omega})= & \frac{1}{2} \operatorname{coth} \frac{\beta_{0} \hat{\omega}}{2} \frac{\Phi^{\mathrm{ig}}(\hat{z}, \hat{\omega})}{\Phi^{\mathrm{ig}(0)}(\hat{\omega})}, \\
C(\hat{z}, \hat{\omega})= & -\frac{1}{1-e^{-\beta_{0} \hat{\omega}}} \frac{\Phi^{\mathrm{ig}}(\hat{z},-\hat{\omega})}{\Phi^{\mathrm{ig}(0)}(-\hat{\omega})} \\
& \times \exp \left\{-\frac{i \hat{\omega}}{\pi T} \arctan (\pi T \hat{z})-\frac{i \hat{\omega}}{2 \pi T} \log (1+\pi T \hat{z})\right\} .
\end{aligned}
$$

While the oscillating factor $(1-\pi T \hat{z})^{i \hat{\omega} /(2 \pi T)}$ is kept unexpanded, it is always legitimate to expand all regular parts, such as $A, B_{1}, C$, in small $\hat{\omega}$. In the small $\hat{\omega}$ limit, the functions $A, B_{1}, C$ and their radial derivatives scale as

$$
\begin{array}{rlrl}
A & =\mathcal{O}\left(\hat{\omega}^{0}\right)+\cdots, & & B_{1}=\mathcal{O}\left(\hat{\omega}^{-1}\right)+\cdots, \\
C & =\mathcal{O}\left(\hat{\omega}^{-1}\right)+\cdots, & \\
\partial_{\hat{z}} A & =\mathcal{O}\left(\hat{\omega}^{1}\right)+\cdots, & \\
\partial_{\hat{z}} C & =\mathcal{O}\left(\hat{\omega}^{0}\right)+\cdots . & &
\end{array}
$$

Before calculating contour integrals (4.19), we make a formal analysis about each integrand. Recall that $A, B_{1}$ are regular, and $B_{2}$ has one branch cut at the horizon. So, in order to have a nonvanishing contribution from each contour integral $I_{i}$ in (4.19), the integrand must contain one $B_{2}$ factor at least. Moreover, since $\partial_{\hat{z}} A \sim \mathcal{O}\left(\hat{\omega}^{1}\right)$, a term containing a factor $\partial_{\hat{z}} A \partial_{\hat{z}} A$ might be of order $\mathcal{O}\left(\hat{\omega}^{2}\right)$.

We take $I_{3}$ as an example to illustrate the computation. In terms of $A, B_{1}, B_{2}$, the integral $I_{3}$ becomes

$$
\begin{aligned}
I_{3}= & \int_{0_{1}}^{0_{2}} d \hat{z} \frac{-i \hat{\omega}_{3} \gamma^{2}}{2 \hat{z}^{2}}\left(1-3 \pi^{2} T^{2} \hat{z}^{2}\right)\left[2 \partial_{\hat{z}} B_{2}\left(\hat{z}_{z}, \hat{\omega}_{1}\right) \partial_{\hat{z}} A\left(\hat{z}, \hat{\omega}_{2}\right) A\left(\hat{z}, \hat{\omega}_{3}\right) \hat{q}_{a}^{L}\left(\hat{\omega}_{1}\right) \hat{q}_{r}^{L}\left(\hat{\omega}_{2}\right) \hat{q}_{r}^{L}\left(\hat{\omega}_{3}\right)\right. \\
& +2 \partial_{\hat{z}} B_{2}\left(\hat{z}, \hat{\omega}_{1}\right) \partial_{\hat{z}} A\left(\hat{z}, \hat{\omega}_{2}\right) B_{1}\left(\hat{z}, \hat{\omega}_{3}\right) \hat{q}_{a}^{L}\left(\hat{\omega}_{1}\right) \hat{q}_{r}^{L}\left(\hat{\omega}_{2}\right) \hat{q}_{a}^{L}\left(\hat{\omega}_{3}\right)+2 \partial_{\hat{z}} B_{2}\left(\hat{z}, \hat{\omega}_{1}\right) \partial_{\hat{z}} B_{1}\left(\hat{z}, \hat{\omega}_{2}\right) A\left(\hat{z}, \hat{\omega}_{3}\right) \hat{q}_{a}^{L}\left(\hat{\omega}_{1}\right) \hat{q}_{a}^{L}\left(\hat{\omega}_{2}\right) \hat{q}_{r}^{L}\left(\hat{\omega}_{3}\right) \\
& +2 \partial_{\hat{z}} B_{2}\left(\hat{z}, \hat{\omega}_{1}\right) \partial_{\hat{z}} B_{1}\left(\hat{z}, \hat{\omega}_{2}\right) B_{1}\left(\hat{z}, \hat{\omega}_{3}\right) \hat{q}_{a}^{L}\left(\hat{\omega}_{1}\right) \hat{q}_{a}^{L}\left(\hat{\omega}_{2}\right) \hat{q}_{a}^{L}\left(\hat{\omega}_{3}\right)+\partial_{z} A\left(\hat{z}, \hat{\omega}_{1}\right) \partial_{z} A\left(\hat{z}, \hat{\omega}_{2}\right) B_{2}\left(\hat{z}, \hat{\omega}_{3}\right) \hat{q}_{r}^{L}\left(\hat{\omega}_{1}\right) \hat{q}_{r}^{L}\left(\hat{\omega}_{2}\right) \hat{q}_{a}^{L}\left(\hat{\omega}_{3}\right) \\
& +\partial_{\hat{z}} B_{1}\left(\hat{z}, \hat{\omega}_{1}\right) \partial_{\hat{z}} B_{1}\left(\hat{z}, \hat{\omega}_{2}\right) B_{2}\left(\hat{z}, \hat{\omega}_{3}\right) \hat{q}_{a}^{L}\left(\hat{\omega}_{1}\right) \hat{q}_{a}^{L}\left(\hat{\omega}_{2}\right) \hat{q}_{a}^{L}\left(\hat{\omega}_{3}\right)+2 \partial_{\hat{z}} A\left(\hat{z}, \hat{\omega}_{1}\right) \partial_{\hat{z}} B_{1}\left(\hat{z}, \hat{\omega}_{2}\right) B_{2}\left(\hat{z}, \hat{\omega}_{3}\right) \hat{q}_{r}^{L}\left(\hat{\omega}_{1}\right) \hat{q}_{a}^{L}\left(\hat{\omega}_{2}\right) \hat{q}_{a}^{L}\left(\hat{\omega}_{3}\right) \\
& +\partial_{\hat{z}} B_{2}\left(\hat{z}, \hat{\omega}_{1}\right) \partial_{\hat{z}} B_{2}\left(\hat{z}, \hat{\omega}_{2}\right) A\left(\hat{z}, \hat{\omega}_{3}\right) \hat{q}_{a}^{L}\left(\hat{\omega}_{1}\right) \hat{q}_{a}^{L}\left(\hat{\omega}_{2}\right) \hat{q}_{r}^{L}\left(\hat{\omega}_{3}\right)+\partial_{\hat{z}} B_{2}\left(\hat{z}, \hat{\omega}_{1}\right) \partial_{\hat{z}} B_{2}\left(\hat{z}, \hat{\omega}_{2}\right) B_{1}\left(\hat{z}, \hat{\omega}_{3}\right) \hat{q}_{a}^{L}\left(\hat{\omega}_{1}\right) \hat{q}_{a}^{L}\left(\hat{\omega}_{2}\right) \hat{q}_{a}^{L}\left(\hat{\omega}_{3}\right) \\
& +2 \partial_{\hat{z}} B_{2}\left(\hat{z}, \hat{\omega}_{1}\right) \partial_{\hat{z}} A\left(\hat{z}, \hat{\omega}_{2}\right) B_{2}\left(\hat{z}, \hat{\omega}_{3}\right) \hat{q}_{a}^{L}\left(\hat{\omega}_{1}\right) \hat{q}_{r}^{L}\left(\hat{\omega}_{2}\right) \hat{q}_{a}^{L}\left(\hat{\omega}_{3}\right)+2 \partial_{\hat{z}} B_{2}\left(\hat{z}_{2}, \hat{\omega}_{1}\right) \partial_{\hat{z}} B_{1}\left(\hat{z}, \hat{\omega}_{2}\right) B_{2}\left(\hat{z}_{3}, \hat{\omega}_{3}\right) \hat{q}_{a}^{L}\left(\hat{\omega}_{1}\right) \hat{q}_{a}^{L}\left(\hat{\omega}_{2}\right) \hat{q}_{a}^{L}\left(\hat{\omega}_{3}\right) \\
& \left.+\partial_{\hat{z}} B_{2}\left(\hat{z}, \hat{\omega}_{1}\right) \partial_{\hat{z}} B_{2}\left(\hat{z}, \hat{\omega}_{2}\right) \partial_{\hat{z}} B_{2}\left(\hat{z}, \hat{\omega}_{3}\right) \hat{q}_{a}^{L}\left(\hat{\omega}_{1}\right) \hat{q}_{a}^{L}\left(\hat{\omega}_{2}\right) \hat{q}_{a}^{L}\left(\hat{\omega}_{3}\right)\right] .
\end{aligned}
$$

Thanks to the delta function $\delta\left(\hat{\omega}_{1}+\hat{\omega}_{2}+\hat{\omega}_{3}\right)$, the last term of (B5) does not contain the singular factor (1$\pi T \hat{z})^{i \hat{\omega} /(2 \pi T)}$ and will be computed via the residue theorem. The rest terms in (B5) contain an overall singular factor $(1-\pi T \hat{z})^{i \hat{\omega} /(2 \pi T)}$ and could be schematically written as

$$
\mathcal{I}=\int_{0_{1}}^{0_{2}} \frac{d \hat{z}}{\hat{z}^{2}}(1-\pi T \hat{z})^{ \pm i \hat{\omega} /(2 \pi T)} \mathcal{H}(\hat{z}, \hat{\omega})
$$

where $\mathcal{H}(\hat{z}, \hat{\omega})$ contains a simple pole and/or a secondorder pole near the horizon. Note that from (B5) to (B6), we 
ignored the purely numerical factor as well as the potential powers of frequency $\hat{\omega}^{m}(m=1$ or 2$)$. Therefore, for the purpose of evaluating $I_{3}$ up to $\mathcal{O}\left(\hat{\omega}^{1}\right)$, it is sufficient to compute (B6) up to $\mathcal{O}\left(\hat{\omega}^{1}\right)$. For a generic function $\mathcal{H}(\hat{z}, \hat{\omega})$ it is challenging to obtain analytical results for (B6). Consider the Laurent expansion of $\mathcal{H}(\hat{z}, \hat{\omega})$ near the horizon

$$
\mathcal{H}(\hat{z}, \hat{\omega})=\sum_{n=-2}^{\infty} \mathcal{H}_{n}(\hat{\omega})(1-\pi T \hat{z})^{n}
$$

which has a convergence radius larger than $1 /(\pi T)$. Therefore, in the domain enclosed by the radial contour, it is legal to represent $\mathcal{H}(\hat{z}, \hat{\omega})$ by its Laurent expansion (B7). Here, in the small frequency limit $\mathcal{H}_{n}(\hat{\omega})$ is a Taylor series of frequency. So, the original task of computing (B6) now boils down to calculating

$$
\begin{gathered}
\mathcal{I}_{n}=\int_{0_{1}}^{0_{2}} \frac{d \hat{z}}{\hat{z}^{2}}(1-\pi T \hat{z})^{ \pm i \hat{\omega} /(2 \pi T)}(1-\pi T \hat{z})^{n}, \\
n=-2,-1,0,1, \ldots,
\end{gathered}
$$

which could be worked out analytically for generic $\hat{\omega}$. Afterwards, we extract the small frequency behavior of $\mathcal{I}_{n}$

$$
\begin{aligned}
& \mathcal{I}_{n}=\mp \hat{\omega} \frac{{ }_{2} F_{1}(2, n+1 ; n+2 ; 1-\Lambda)}{(n+1) T}+\mathcal{O}\left(\hat{\omega}^{2}\right), \quad n \geq 1, \\
& \mathcal{I}_{n}=\mp \frac{\hat{\omega}}{\Lambda T}+\mathcal{O}\left(\hat{\omega}^{2}\right), \quad n=0, \\
& \mathcal{I}_{n}=2 i \pi \mp \frac{\hat{\omega}}{\Lambda T}+\mathcal{O}\left(\hat{\omega}^{2}\right), \quad n=-1, \\
& \mathcal{I}_{n}=4 i \pi+\mathcal{O}(\hat{\omega}), \quad n=-2,
\end{aligned}
$$

where $\Lambda$ represents a UV cutoff near the AdS boundary. It is straightforward to check that the results (B9) could be correctly obtained by first expanding the integrand of (B8) in small $\hat{\omega}$ and then doing the radial integral. However, this latter approach cannot correctly cover higher order terms omitted in (B9), which correspond to higher order terms in the SK action.

Now, we turn to the $\operatorname{arr}$ terms at the order $\mathcal{O}\left(\hat{\omega}^{2}\right)$, which correspond to the first line and fifth line in (B5). With the scaling (B4), it is obvious that only the first term of (B5) is relevant. Moreover, only the lowest order results in (B9) are required.

The main lesson from analysis above is as follows. In order to obtain SK effective action to the order $\mathcal{O}\left(\hat{\omega}^{1}\right)$, it is legal although accidental to first expand the integrands [including $(1-\pi T \hat{z})^{ \pm i \hat{\omega} /(2 \pi T)}$ ] in (4.19) in small $\hat{\omega}$, and then implement the radial integral. While generically this might not be true for higher order terms, it does work for extracting arr terms at the order $\mathcal{O}\left(\hat{\omega}^{2}\right)$.

\section{APPENDIX C: KMS CONDITIONS FOR THREE-POINT FUNCTIONS}

Consider a quantum many-body system in a thermal state, which also preserves $\mathcal{P} \mathcal{T}$ symmetry. The KMS condition, satisfied by the generating functional $W$, sets constraints among three-point functions [57]. These constraints were also rederived and extended to a situation with multiple variables in [17]. In this Appendix, we review these constraints for completeness. Here, we closely follow Sec. II D of [17].

At cubic level, the generating functional can be written as

$$
\begin{aligned}
W_{\mathrm{cub}}= & i\left[\frac{1}{3 !} G^{l m n} \phi_{a}^{l} \phi_{a}^{m} \phi_{a}^{n}+\frac{i}{2} H^{l m n} \phi_{a}^{l} \phi_{a}^{m} \phi_{r}^{n}\right. \\
& \left.+\frac{1}{2} K^{l m n} \phi_{a}^{l} \phi_{r}^{m} \phi_{r}^{n}\right]
\end{aligned}
$$

where the indices $l$, etc., label different bosonic Hermitian operators $\mathcal{O}^{l}$ and associated sources $\phi^{l}$. Each term in (C1) should be understood as a convolution in Fourier space, e.g.,

$$
\begin{aligned}
G^{l m n} & \phi_{a}^{l} \phi_{a}^{m} \phi_{a}^{n} \\
= & \int \frac{d \omega_{2} d \omega_{3}}{(2 \pi)^{2}} G^{l m n}\left(\omega_{1}, \omega_{2}, \omega_{3}\right) \phi_{a}^{l}\left(\omega_{1}\right) \phi_{a}^{m}\left(\omega_{2}\right) \phi_{a}^{n}\left(\omega_{3}\right), \\
& \omega_{1}+\omega_{2}+\omega_{3}=0,
\end{aligned}
$$

where spatial momenta are suppressed. The three-point functions $G_{\alpha_{1} \alpha_{2} \alpha_{3}}^{l m n}$, with $\alpha_{1,2,3}=r$ or $a$, are third order functional derivatives of $W_{\text {cub }}$ with respect to sources $\phi_{r, a}^{l}$. Then, we have

$$
G^{l m n}=-G_{r r r}^{l m n}, \quad H^{l m n}=G_{r r a}^{l m n}, \quad K^{l m n}=G_{r a a}^{l m n} .
$$

To present KMS constraints for three-point functions, it is convenient to introduce the following notations:

$$
\begin{aligned}
H_{3}^{l m n} \equiv G_{r r a}^{l m n}, & H_{2}^{l m n} \equiv G_{r a r}^{l m n}, & H_{1}^{l m n} \equiv G_{a r r}^{l m n}, \\
K_{1}^{l m n} \equiv G_{r a a}^{l m n}, & K_{2}^{l m n} \equiv G_{\text {ara }}^{l m n}, & K_{3}^{l m n} \equiv G_{\text {aar }}^{l m n} .
\end{aligned}
$$

Note that in (C3) and (C4) variables $\omega$ 's are further suppressed. For a $\mathcal{P} \mathcal{T}$-invariant theory, the KMS condition applied to three-point functions implies

$$
\begin{aligned}
H_{1}= & \frac{i}{2}\left(N_{3}+N_{2}\right) K_{1}^{*}-\frac{i}{2}\left(N_{2} K_{3}+N_{3} K_{2}\right), \\
H_{2}= & \frac{i}{2}\left(N_{3}+N_{1}\right) K_{2}^{*}-\frac{i}{2}\left(N_{1} K_{3}+N_{3} K_{1}\right), \\
H_{3}= & \frac{i}{2}\left(N_{1}+N_{2}\right) K_{3}^{*}-\frac{i}{2}\left(N_{1} K_{2}+N_{2} K_{1}\right), \\
G= & \frac{1}{4}\left[\left(K_{1}^{*}+K_{2}^{*}+K_{3}^{*}\right)+2 N_{2} N_{3} \operatorname{Re} K_{1}+2 N_{1} N_{3} \operatorname{Re} K_{2}\right. \\
& \left.+2 N_{1} N_{2} \operatorname{Re} K_{3}\right],
\end{aligned}
$$


where for brevity the indices $l, m, n$ in the functions (C3) and $(\mathrm{C} 4)$ are suppressed. Moreover, we introduced

$$
N_{a} \equiv \operatorname{coth} \frac{\beta_{0} \omega_{a}}{2}, \quad a=1,2,3
$$

The first three equations in (C5) satisfy permutation symmetry among indices (123).
In order to guarantee regularity in the limit $\omega \rightarrow 0$, the first three of (C5) will involve some terms in (C1) with at least one time derivative, while the last of (C5) will involve some terms in $(\mathrm{C} 1)$ with at least two time derivatives. This explains why we need to additionally track arr-type terms in (4.36) at $\mathcal{O}\left(\hat{\omega}^{2}\right)$.
[1] E. A. Calzetta and B.-L. B. Hu, Nonequilibrium Quantum Field Theory (Cambridge University Press, Cambridge, England, 2009).

[2] A. Kamenev, Field Theory of Non-Equilibrium Systems (Cambridge University Press, Cambridge, England, 2011).

[3] A. O. Caldeira and A. J. Leggett, Path integral approach to quantum Brownian motion, Physica (Amsterdam) 121A, 587 (1983).

[4] R. P. Feynman and F. L. Vernon, Jr., The theory of a general quantum system interacting with a linear dissipative system, Ann. Phys. (N.Y.) 24, 118 (1963).

[5] D. T. Son and D. Teaney, Thermal noise and stochastic strings in AdS/CFT, J. High Energy Phys. 07 (2009) 021.

[6] Y. Akamatsu, Real-time quantum dynamics of heavy quark systems at high temperature, Phys. Rev. D 87, 045016 (2013).

[7] L. V. Keldysh, Diagram technique for nonequilibrium processes, Zh. Eksp. Teor. Fiz. 47, 1515 (1964).

[8] J. S. Schwinger, Brownian motion of a quantum oscillator, J. Math. Phys. (N.Y.) 2, 407 (1961).

[9] K.-c. Chou, Z.-b. Su, B.-1. Hao, and L. Yu, Equilibrium and nonequilibrium formalisms made unified, Phys. Rep. 118, 1 (1985).

[10] B. Chakrabarty, S. Chaudhuri, and R. Loganayagam, Out of time ordered quantum dissipation, J. High Energy Phys. 07 (2019) 102.

[11] B. Chakrabarty and S. Chaudhuri, Out of time ordered effective dynamics of a quartic oscillator, SciPost Phys. 7, 013 (2019).

[12] S. Endlich, A. Nicolis, R. A. Porto, and J. Wang, Dissipation in the effective field theory for hydrodynamics: First order effects, Phys. Rev. D 88, 105001 (2013).

[13] P. Kovtun, G. D. Moore, and P. Romatschke, Towards an effective action for relativistic dissipative hydrodynamics, J. High Energy Phys. 07 (2014) 123.

[14] A. Nicolis, R. Penco, and R. A. Rosen, Relativistic fluids, superfluids, solids and supersolids from a coset construction, Phys. Rev. D 89, 045002 (2014).

[15] M. Harder, P. Kovtun, and A. Ritz, On thermal fluctuations and the generating functional in relativistic hydrodynamics, J. High Energy Phys. 07 (2015) 025.

[16] S. Grozdanov and J. Polonyi, Viscosity and dissipative hydrodynamics from effective field theory, Phys. Rev. D 91, 105031 (2015).

[17] M. Crossley, P. Glorioso, and H. Liu, Effective field theory of dissipative fluids, J. High Energy Phys. 09 (2017) 095.
[18] P. Glorioso, M. Crossley, and H. Liu, Effective field theory of dissipative fluids (II): Classical limit, dynamical KMS symmetry and entropy current, J. High Energy Phys. 09 (2017) 096.

[19] F. M. Haehl, R. Loganayagam, and M. Rangamani, The fluid manifesto: Emergent symmetries, hydrodynamics, and black holes, J. High Energy Phys. 01 (2016) 184.

[20] F. M. Haehl, R. Loganayagam, and M. Rangamani, Topological sigma models \& dissipative hydrodynamics, J. High Energy Phys. 04 (2016) 039.

[21] F. M. Haehl, R. Loganayagam, and M. Rangamani, Effective action for relativistic hydrodynamics: Fluctuations, dissipation, and entropy inflow, J. High Energy Phys. 10 (2018) 194.

[22] K. Jensen, N. Pinzani-Fokeeva, and A. Yarom, Dissipative hydrodynamics in superspace, J. High Energy Phys. 09 (2018) 127.

[23] X. Chen-Lin, L. V. Delacretaz, and S. A. Hartnoll, Theory of Diffusive Fluctuations, Phys. Rev. Lett. 122, 091602 (2019).

[24] M. Baggioli and M. Landry, Effective field theory for quasicrystals and phasons dynamics, SciPost Phys. 9, 062 (2020).

[25] A. Jain, P. Kovtun, A. Ritz, and A. Shukla, Hydrodynamic effective field theory and the analyticity of hydrostatic correlators, J. High Energy Phys. 02 (2021) 200.

[26] H. Liu and P. Glorioso, Lectures on non-equilibrium effective field theories and fluctuating hydrodynamics, Proc. Sci., TASI2017 (2018) 008.

[27] J. M. Maldacena, The Large N limit of superconformal field theories and supergravity, Int. J. Theor. Phys. 38, 1113 (1999).

[28] S. Gubser, I. R. Klebanov, and A. M. Polyakov, Gauge theory correlators from noncritical string theory, Phys. Lett. B 428, 105 (1998).

[29] E. Witten, Anti-de Sitter space and holography, Adv. Theor. Math. Phys. 2, 253 (1998).

[30] C. P. Herzog, A. Karch, P. Kovtun, C. Kozcaz, and L. G. Yaffe, Energy loss of a heavy quark moving through $N=4$ supersymmetric Yang-Mills plasma, J. High Energy Phys. 07 (2006) 013.

[31] J. Casalderrey-Solana and D. Teaney, Heavy quark diffusion in strongly coupled $N=4$ Yang-Mills, Phys. Rev. D 74, 085012 (2006).

[32] S. S. Gubser, Drag force in AdS/CFT, Phys. Rev. D 74, 126005 (2006). 
[33] J. Casalderrey-Solana and D. Teaney, Transverse momentum broadening of a fast quark in a $N=4$ Yang Mills plasma, J. High Energy Phys. 04 (2007) 039.

[34] S. S. Gubser, Momentum fluctuations of heavy quarks in the gauge-string duality, Nucl. Phys. B790, 175 (2008).

[35] K. Murata, Fast scrambling in holographic Einstein-Podolsky-Rosen pair, J. High Energy Phys. 11 (2017) 049.

[36] J. de Boer, E. Llabrés, J. F. Pedraza, and D. Vegh, Chaotic Strings in AdS/CFT, Phys. Rev. Lett. 120, 201604 (2018).

[37] R.-G. Cai, S.-M. Ruan, R.-Q. Yang, and Y.-L. Zhang, The string worldsheet as the holographic dual of SYK state, arXiv: 1709.06297.

[38] J. de Boer, V. E. Hubeny, M. Rangamani, and M. Shigemori, Brownian motion in AdS/CFT, J. High Energy Phys. 07 (2009) 094.

[39] C. Herzog and D. Son, Schwinger-Keldysh propagators from AdS/CFT correspondence, J. High Energy Phys. 03 (2003) 046.

[40] D. T. Son and A. O. Starinets, Minkowski space correlators in AdS/CFT correspondence: Recipe and applications, J. High Energy Phys. 09 (2002) 042.

[41] G. C. Giecold, E. Iancu, and A. H. Mueller, Stochastic trailing string and Langevin dynamics from AdS/CFT, J. High Energy Phys. 07 (2009) 033.

[42] J. Casalderrey-Solana, K.-Y. Kim, and D. Teaney, Stochastic string motion above and below the world sheet horizon, J. High Energy Phys. 12 (2009) 066.

[43] S. Caron-Huot, P. M. Chesler, and D. Teaney, Fluctuation, dissipation, and thermalization in non-equilibrium AdS_5 black hole geometries, Phys. Rev. D 84, 026012 (2011).

[44] B. Chakrabarty, J. Chakravarty, S. Chaudhuri, C. Jana, R. Loganayagam, and A. Sivakumar, Nonlinear Langevin dynamics via holography, J. High Energy Phys. 01 (2020) 165.

[45] P. Glorioso, M. Crossley, and H. Liu, A prescription for holographic Schwinger-Keldysh contour in non-equilibrium systems. arXiv:1812.08785.

[46] K. Skenderis and B. C. van Rees, Real-time gauge/gravity duality, Phys. Rev. Lett. 101, 081601 (2008).

[47] K. Skenderis and B. C. van Rees, Real-time gauge/gravity duality: Prescription, renormalization and examples, J. High Energy Phys. 05 (2009) 085.

[48] J. de Boer, M. P. Heller, and N. Pinzani-Fokeeva, Holographic Schwinger-Keldysh effective field theories, J. High Energy Phys. 05 (2019) 188.

[49] C. Jana, R. Loganayagam, and M. Rangamani, Open quantum systems and Schwinger-Keldysh holograms, J. High Energy Phys. 07 (2020) 242.

[50] B. Chakrabarty and P. Aswin, Open effective theory of scalar field in rotating plasma, arXiv:2011.13223.

[51] R. Loganayagam, K. Ray, and A. Sivakumar, Fermionic open EFT from holography, arXiv:2011.07039.

[52] R. Loganayagam, K. Ray, S. K. Sharma, and A. Sivakumar, Holographic KMS relations at finite density, J. High Energy Phys. 03 (2021) 233.

[53] J. K. Ghosh, R. Loganayagam, S. G. Prabhu, M. Rangamani, A. Sivakumar, and V. Vishal, Effective field theory of stochastic diffusion from gravity, J. High Energy Phys. 05 (2021) 130.
[54] Y. Bu, T. Demircik, and M. Lublinsky, All order effective action for charge diffusion from Schwinger-Keldysh holography, J. High Energy Phys. 05 (2021) 187.

[55] Y. Bu, M. Fujita, and S. Lin, Ginzburg-Landau effective action for a fluctuating holographic superconductor, arXiv: 2106.00556.

[56] T. He, R. Loganayagam, M. Rangamani, and J. Virrueta, An effective description of momentum diffusion in a charged plasma from holography, arXiv:2108.03244.

[57] E. Wang and U.W. Heinz, A generalized fluctuation dissipation theorem for nonlinear response functions, Phys. Rev. D 66, 025008 (2002).

[58] M. Crossley, P. Glorioso, H. Liu, and Y. Wang, Off-shell hydrodynamics from holography, J. High Energy Phys. 02 (2016) 124.

[59] U. Gursoy, E. Kiritsis, L. Mazzanti, and F. Nitti, Langevin diffusion of heavy quarks in non-conformal holographic backgrounds, J. High Energy Phys. 12 (2010) 088.

[60] N. Sogabe, N. Yamamoto, and Y. Yin, Positive magnetoresistance induced by hydrodynamic fluctuations in chiral media, arXiv:2105.10271.

[61] C. Jana, A study of non-linear Langevin dynamics under non-Gaussian noise with quartic cumulant, arXiv:2108 .04284 .

[62] N. Abbasi and A. Davody, Moving quark in a viscous fluid, J. High Energy Phys. 06 (2012) 065.

[63] N. Abbasi and A. Davody, The energy loss of a heavy quark moving through a general fluid dynamical flow, J. High Energy Phys. 12 (2013) 026.

[64] M. Lekaveckas and K. Rajagopal, Effects of fluid velocity gradients on heavy quark energy loss, J. High Energy Phys. 02 (2014) 068.

[65] J. Reiten and A. V. Sadofyev, Drag force to all orders in gradients, J. High Energy Phys. 07 (2020) 146.

[66] S. Bhattacharyya, V.E. Hubeny, S. Minwalla, and M. Rangamani, Nonlinear fluid dynamics from gravity, J. High Energy Phys. 02 (2008) 045.

[67] P. M. Chesler, M. Lekaveckas, and K. Rajagopal, Heavy quark energy loss far from equilibrium in a strongly coupled collision, J. High Energy Phys. 10 (2013) 013.

[68] J.-T. Hsiang and B.-L. Hu, Fluctuation-dissipation relation from the nonequilibrium dynamics of a nonlinear open quantum system, Phys. Rev. D 101, 125003 (2020).

[69] U. Gursoy, E. Kiritsis, G. Michalogiorgakis, and F. Nitti, Thermal transport and drag force in improved holographic QCD, J. High Energy Phys. 12 (2009) 056.

[70] U. Gursoy, E. Kiritsis, L. Mazzanti, G. Michalogiorgakis, and F. Nitti, Improved holographic QCD, Lect. Notes Phys. 828, 79 (2011).

[71] R.-G. Cai, S. He, and D. Li, A hQCD model and its phase diagram in Einstein-Maxwell-Dilaton system, J. High Energy Phys. 03 (2012) 033.

[72] R.-G. Cai, S. Chakrabortty, S. He, and L. Li, Some aspects of QGP phase in a hQCD model, J. High Energy Phys. 02 (2013) 068.

[73] M. Chernicoff, D. Fernandez, D. Mateos, and D. Trancanelli, Drag force in a strongly coupled anisotropic plasma, J. High Energy Phys. 08 (2012) 100. 
[74] D. Giataganas and H. Soltanpanahi, Heavy quark diffusion in strongly coupled anisotropic plasmas, J. High Energy Phys. 06 (2014) 047.

[75] L. Cheng, X.-H. Ge, and S.-Y. Wu, Drag force of Anisotropic plasma at finite $U(1)$ chemical potential, Eur. Phys. J. C 76, 256 (2016).

[76] V. Jahnke and A. S. Misobuchi, Probing strongly coupled anisotropic plasmas from higher curvature gravity, Eur. Phys. J. C 76, 309 (2016).

[77] K. Chelabi, Z. Fang, M. Huang, D. Li, and Y.-L. Wu, Chiral phase transition in the soft-wall model of AdS/QCD, J. High Energy Phys. 04 (2016) 036.

[78] R. Rougemont, A. Ficnar, S. Finazzo, and J. Noronha, Energy loss, equilibration, and thermodynamics of a baryon rich strongly coupled quark-gluon plasma, J. High Energy Phys. 04 (2016) 102.

[79] S. Li, K. A. Mamo, and H.-U. Yee, Jet quenching parameter of the quark-gluon plasma in a strong magnetic field:
Perturbative QCD and AdS/CFT correspondence, Phys. Rev. D 94, 085016 (2016).

[80] Z.-q. Zhang, K. Ma, and D.-f. Hou, Drag force in strongly coupled supersymmetric Yang-Mills plasma in a magnetic field, J. Phys. G 45, 025003 (2018).

[81] X. Chen, D. Li, D. Hou, and M. Huang, Quarkyonic phase from quenched dynamical holographic QCD model, J. High Energy Phys. 03 (2020) 073.

[82] I. Y. Aref'eva, K. Rannu, and P. Slepov, Energy loss in holographic anisotropic model for heavy quarks in external magnetic field, arXiv:2012.05758.

[83] J. Sonner and A. G. Green, Hawking Radiation and Nonequilibrium Quantum Critical Current Noise, Phys. Rev. Lett. 109, 091601 (2012).

[84] F. Bigazzi, A. Caddeo, T. Canneti, and A. L. Cotrone, Bubble wall velocity at strong coupling, arXiv:2104 .12817 . 